\title{
Féeries
}

Études sur le conte merveilleuX, XVII ${ }^{e}$ XIXe siècle

9 | 2012

Le dialogisme intertextuel des contes des Grimm

\section{Les commentaires paratextuels des Kinder- und Hausmärchen, gesammelt durch die Brüder Grimm}

The paratextual commentaries of the Kinder-und Hausmärchen, gesammelt durch die Brüder Grimm

Loreto Núñez

\section{OpenEdition}

\section{Journals}

Édition électronique

URL : http://journals.openedition.org/feeries/829

DOI : $10.4000 /$ feeries.829

ISSN : $1957-7753$

Éditeur

UGA Éditions/Université Grenoble Alpes

Édition imprimée

Date de publication : 15 octobre 2012

Pagination : 197-247

ISBN : 978-2-84310-233-2

ISSN : $1766-2842$

\section{Référence électronique}

Loreto Núñez, «Les commentaires paratextuels des Kinder- und Hausmärchen, gesammelt durch die Brüder Grimm », Féeries [En ligne], 9 | 2012, mis en ligne le 15 octobre 2013, consulté le 07 septembre 2020. URL : http://journals.openedition.org/feeries/829 ; DOI : https://doi.org/10.4000/feeries.829 
Centre de recherche en langues et littératures européennes comparées (CLE),

Université de Lausanne

\author{
LES COMMENTAIRES PARATEXTUELS DES \\ KINDER- UND HAUSMÄRCHEN, GESAMMELT DURCH \\ DIE BRÜDER GRIMM
}

A Consuelo Ruiz Montero, una amiga, también de los Grimm.

$L$ A LECTURE ET SURTOUT L'ÉTUDE de l'œuvre des Grimm et de leurs Kinder- und Hausmärchen en particulier, procurent souvent l'impression qu'on est seulement devant la partie émergée d'un iceberg et qu'une découverte en cache beaucoup d'autres. Les considérations présentées ici ne mettront à jour que la pointe de cet iceberg et elles n'auront d'autre ambition que d'inviter à l'analyse détaillée de parties moins étudiées des Kinder- und Hausmärchen (KHM) : les commentaires paratextuels (préfaces, annotations, considérations sur la littérature, témoignages). Il est nécessaire de céder la parole aux Grimm eux-mêmes et c'est ce que je m'efforcerai de faire le plus possible dans cette contribution, car ils nous ont donné, peut-être plus que d'autres auteurs, de nombreuses et précieuses informations sur leur œuvre.

Je développerai ici la même thèse qu'Ute Heidmann, à savoir que ces renseignements nous sont offerts dans le but stratégique de créer une certaine image des Kinder- und Hausmärchen ou, pour être plus précise, d'en faire miroiter des facettes bien particulières. Comme il serait trop tôt pour proposer une démonstration conclusive et générale, je me limiterai ici à présenter, à partir des "Témoignages» ("Zeugnisse»), quelques hypothèses accompagnées de réflexions les nuançant. Ces considérations seront suivies par une traduction de travail des premiers chapitres de la section "Littérature» ("Literatur»). Si les «annotations»( "Anmerkungen») sont tout aussi importantes pour une étude sérieuse des KHM, les sections «Témoignages» et «Littérature» offrent l'avantage d'être plus générales car, 
n'étant pas concentrées sur un conte en particulier, elles donnent ainsi la possibilité de dresser un panorama plus vaste du propos général des Grimm. Le but de cette contribution est de donner à la recherche de langue française des documents qui nous semblent importants, en dépit des difficultés de traduction que présentent ces notes des Grimm, souvent allusives et rédigées dans un style beaucoup moins soigné que les contes eux-mêmes.

\section{Quelques questions à partir des "Témoignages» («Zeugnisse»)}

L’importance des «Témoignages» ("Zeugnisse») se perçoit déjà dans l'augmentation de leur nombre tout au long des années. En effet, dans la première édition de I8I2/I8I5 ils ne sont encore qu'au nombre de six, alors que ce chiffre passe à trente-sept dans la dernière édition parue du vivant des Grimm. Cet accroissement s'accompagne d'un double élargissement, temporel et linguistique. En effet, si dans la première édition on ne trouvait qu'un auteur antique (Strabon), ultérieurement les témoignages provenant de l'Antiquité passent à six, incluant à côté des exemples grecs des exemples latins. Le Moyen Âge, qui était absent de la première édition, prend également une place importante. Deux autres types d'ajouts méritent d'être mentionnés : d'une part, l'augmentation des références à des auteurs contemporains des Grimm, y compris à des personnes ayant recensé les KHM, qui deviennent alors des cautions de l'ouvrage; de l'autre, l'inclusion de trois témoignages en espagnol. Les «Témoignages» ont subi une autre modification qu'il convient de regarder de plus près : le changement de leur place au sein des KHM.

\section{Le transfert des "Témoignages» ("Zeugnisse») au sein des KHM comme signe d'un déplacement de l'explicitation de l'intertextualité}

Dans les premières éditions des KHM, la section «Témoignages» apparaît juste après la préface. Elle précède le corpus des contes qui, à son tour, est suivi des annotations. Cette structure change en I822, quand les Grimm publient un troisième volume contenant les annotations, les fragments, les ajouts de témoignages et de réflexions sur la littérature existante. Ces modifications de structure poursuivent, selon moi, deux buts qui pourraient paraître contradictoires mais que les Grimm semblent avoir réalisés à travers la nouvelle constellation : tout en démarquant leurs propres Märchen, ils conservent ce que nous appelons intertextualité en la déplaçant et la "refonctionnalisant». Alors que dans la première édition, les "Zeugnisse» devaient consolider et asseoir leur projet, la situation a changé lors de la publication du troisième volume avec paratextes : l'idée du projet s'est 
réalisée et a été acceptée; à présent, les "Zeugnisse» et les autres sections paratextuelles servent à consolider le projet des Grimm. En même temps, toutes ces informations sont présentées comme relevant de ce qu'ils désignent comme un discours scientifique à destination d'un public restreint. La préface de l'édition de I837 me semble aller dans ce sens, quand les Grimm expliquent le détachement de la troisième partie :

Der dritte Teil, dessen Inhalt sich lediglich auf den wissenschaftlichen Gebrauch der Sammlung bezieht und daher nur in einem viel engern Kreis Eingang finden konnte, ist diesmal nicht mit abgedruckt [...]. In der Folge soll dieser dritte Teil als ein für sich bestehendes Werk erscheinen, in welchem auch die in der vorigen Ausgabe vorangesetzten Einleitungen von dem Wesen der Märchen und von Kindersitten einen Platz finden werden.

La troisième partie, dont le contenu se réfère uniquement à l'usage scientifique du recueil et qui pour cela n'a touché qu'un cercle beaucoup plus limité, n’est cette fois pas imprimée ici [...]. Dans la suite, ce troisième volume devra paraître comme une œuvre en elle-même, dans laquelle trouveront aussi place les introductions sur l'essence des contes et les mœurs enfantines, qui, dans l'édition précédente, étaient placées au début ${ }^{ }$.

Or, cette «scientificité» s'avère être, comme les contes eux-mêmes et la scénographie étudiée dans ce volume par Ute Heidmann, une construction, voire un leurre. Il s'agit plutôt d'un discours pseudo-scientifique. En effet, force est de constater que le discours scientifique des Grimm contient plusieurs erreurs ou inexactitudes (que je mentionnerai au fur et à mesure), voire des manipulations.

\section{Les «Zeugnisse » présentent-ils les KHM comme l'aboutissement d'une évolution chronologique?}

Si l'on s'attarde sur la datation des témoignages, on voit qu'ils sont présentés, dans les grandes lignes, selon une suite chronologique. On commence par l'Antiquité classique, puis l'époque de l'empire romain, pour enchaîner sur l'Antiquité tardive; vient ensuite le Moyen Âge, avec la mention de

I. J. et W. Grimm, KHM, I837, préface, p. XxiII / H. Rölleke, KHM, 20Io, I, p. 24. Dans cette contribution, les références des citations tirées des KHM renvoient simultanément aux éditions correspondantes des Grimm ainsi qu'à l'édition de $\mathrm{H}$. Rölleke dont les volumes sont signalés en chiffres romains. J'indique également le volume des autres ouvrages cités. Par ailleurs, comme mon texte inclut de nombreuses citations, on trouvera toutes les références en notes pour faciliter la lecture. Les traductions de citations en langues étrangères sont miennes, sauf mention particulière; pour les modalités de traduction, je me permets de renvoyer aux remarques initiales de la deuxième partie de cette contribution. J'aimerais remercier ici Ute Heidmann de m’avoir encouragée à me lancer dans cette merveilleuse aventure et de m'avoir aidée de ses commentaires. Mes remerciements vont aussi à Jean-Michel Adam pour sa relecture attentive de mon texte et à Anne Defrance pour ses suggestions très pertinentes. 
textes allemands du XIII ${ }^{\mathrm{e}}$ siècle et du XIV ${ }^{\mathrm{e}}$ siècle; suit la citation d'auteurs des $\mathrm{XVI}^{\mathrm{e}}$ et XVII ${ }^{\mathrm{e}}$ siècles et, finalement, sont mentionnés les témoignages du $\mathrm{XVIII}^{\mathrm{e}}$ et XIX ${ }^{\mathrm{e}}$ siècles, qui se rapprochent de plus en plus des Grimm, pour s'achever sur des textes contemporains.

Sennewald a proposé une explication de cette présentation chronologique : elle placerait dans une suite temporelle Dorothea Viehmann, dont les Grimm affirment avoir appris de nombreux contes; en même temps, cet emplacement lui donnerait une confirmation historique et de ce fait une légitimation ${ }^{2}$. Peut-être peut-on aller plus loin et étendre cette proposition aux KHM, dans la mesure où ils sont ainsi présentés comme la continuation, voire l'aboutissement d'une évolution chronologique? En effet, les trois derniers témoignages ne sont pas seulement contemporains des Grimm, mais ils mentionnent aussi leur ouvrage et cela, dans une gradation positive. Le témoignage $\mathrm{n}^{\circ} 35$ cite un article de $\mathrm{I} 8 \mathrm{I} 9$ de Francis Cohen :

Kinder- und Hausmärchen, a collection of German popular stories singular in its kind, both for extent and variety, an [d] from which we have acquired much information.

Kinder-und Hausmärchen, une collection d'histoires populaires allemandes singulière en son genre, aussi bien pour son étendue que pour sa variété, et dont nous avons tiré beaucoup d'informations ${ }^{3}$.

L'importance que les Grimm accordent à cette critique positive, bien qu'ils ne la citent ici que brièvement, se perçoit dans le fait qu'elle est mentionnée dans une note de la préface de I837 :

Nachdem Francis Cohen im Quarterly Review (r819 Mai) die ältere Ausgabe ausführlich angezeigt hatte, erschien nach der zweiten eine Übersetzung von Taylor [...]

Après que Francis Cohen a eu notifié de façon détaillée la plus ancienne édition, une traduction de Taylor est parue après la deuxième $[\ldots]^{4}$

Suit une liste de traductions des KHM qui montre non seulement la réception positive de l'œuvre des Grimm, mais aussi sa diffusion. Si nous revenons sur le témoignage que reprend cette note, nous constatons qu'il n'est pas question des Grimm, mais seulement de leur œuvre, par la mention du titre Kinder-und Hausmärchen. La situation change dans le témoignage $\mathrm{n}^{\circ} 36$, un article paru en I82I et dont l'auteur n'est pas indiqué. Je n'en cite qu'un extrait :

2. J. E. Sennewald, Das Buch, das wir sind, 2004, p. $5 \mathrm{I}$.

3. J. et W. Grimm, KHM, I856, p. 28I / H. Rölleke, KHM, 20IO, III, p. 305.

4. J. et W. Grimm, KHM, I837, préface, p. Xxiır n.* / H. Rölleke, KHM, 20Io, I, p. 24. 
[...] we may also notice the beautiful collection of Nursery Literature, which has lately been edited with so much care by Messrs. Grimm.

[...] nous pourrions aussi noter la splendide collection de littérature de nourrice qui vient d'être éditée avec beaucoup de soin par Messieurs Grimms.

On voit que la critique est tout aussi positive que dans le témoignage précédent, mais que cette fois-ci, les Grimm sont nommés explicitement. Cependant, faut-il ajouter qu'ils ne sont pas vraiment mentionnés comme auteurs, mais comme éditeurs, voire collecteurs? Cela correspond bien sûr à l'image que les Grimm ont donnée d'eux-mêmes, notamment déjà dans la préface de la première édition qui a précédé la recension, comme le montre ici-même Ute Heidmann. Or, en citant le compte rendu, ils l'utilisent à leur tour pour consolider l'image qu'ils ont eux-mêmes créée.

\section{Le choix des sphères linguistiques des témoignages ("Zeugnisse ») : l'interculturalité complexe des Märchen}

Ces deux témoignages évoquent de plus la culture, voire même la «nationalité» à laquelle sont rattachés les contes à travers les "Zeugnisse». Dans le témoignage $\mathrm{n}^{\circ} 35$, nous l'avons vu, on parle "d'histoires populaires allemandes » (German popular stories ${ }^{6}$ ). Au début du témoignage $\mathrm{n}^{\circ} 36$, on lit :

Among the most venerable remains of ancient teutonic literature we should rank the abundant stores of popular legends and traditions.

Parmi les vestiges les plus vénérables de l'ancienne littérature teutonne, nous devrions compter les stocks abondants de légendes et traditions populaires ${ }^{7}$.

Deux autres témoignages établissent un lien entre les contes et la culture allemande. Le n ${ }^{\circ}$ I5, citant les Fabulae Aesopicae de Joachim Camerarius, commence par ces mots :

Hoc autem fabularum genus quale sit, optime poterit intelligi exemplo et comparatione veterum fabularum nationis et genis teutonicae.

Mais la nature de ce genre de fables peut être mieux comprise à travers l'exemple et la comparaison des vieilles fables de la nation et du peuple teuton ${ }^{8}$.

L'auteur du témoignage ${ }^{\circ} 37$, au sujet duquel les Grimm n’indiquent que les initiales C.S., tout en acceptant l'existence d'une transmission orale de

5. J. et W. Grimm, KHM, I856, p. 282 / H. Rölleke, KHM, 20IO, III, p. 305.

6. Ibid., p. 28I / Ibid.

7. Ibid. / Ibid.

8. Ibid., p. 275 / Ibid., p. 299. 
contes dans le Languedoc et la Provence, va jusqu’à affirmer que «la France n'a point comme l'Allemagne et l'Italie une littérature populaire écrite ${ }^{9}$.

Si dans les «Témoignages», plusieurs langues comme le grec, le latin, l'espagnol, le français et l'anglais sont citées à côté de l'allemand, c'est uniquement avec la culture allemande que les contes sont expressément mis en relation. Or, ce n'est pas exactement ce qu'annonce la préface du troisième volume :

Die zusammengestellten Zeugnisse bestätigen das Dasein der Märchen in verschiedenen Zeiten und bei verschiedenen Völkern.

Les témoignages réunis confirment la présence des contes à différentes époques et auprès de différents peuples ${ }^{10}$.

Ce qui est frappant, c'est que ces affirmations sur l'origine nationale des contes ne sont jamais effectuées par des sources de langue allemande, mais toujours étrangères. Je pense que cela peut être perçu comme le miroir du procédé des Grimm : tout comme ce ne sont pas ici eux qui établissent le lien entre la culture allemande et le conte, mais les témoins qu'ils citent, ce n'est pas la langue allemande qui affirme son lien avec le conte, mais ce sont les autres langues.

En effet, les citations de l'allemand se limitent à démontrer (sans la commenter) l'existence du conte dans cette culture. Or, cette existence est de longue date, puisqu'elle est déjà présente dans l'ancien allemand, comme le montrent la citation de la chanson de Gudrun ( $\left.n^{\circ} 9\right)$ et les allusions médiévales qui la suivent. Cette ancienneté va même au-delà, en étant présentée comme faisant partie de l'histoire du conte. En effet, l'extrait tiré de la chanson de Gudrun qui parle du "conte» (Mare) de la montagne aimantée est le tout premier exemple qui ne soit ni en grec ni en latin, comme si après le conte antique venait immédiatement le conte allemand. Cette stratégie de germanisation par "délégation " prend appui sur un passage de la préface de I837 :

Der wissenschaftliche Wert dieser Überlieferungen hat sich in mancher überraschenden Verwandtschaft mit alten Göttersagen bewährt, und die deutsche Mythologie nicht selten Gelegenheit gehabt darauf zurückzukommen, ja sie hat in der Übereinstimmung mit nordischen Mythen einen Beweis des ursprünglichen Zusammenhangs gefunden.

La valeur scientifique de cette tradition a fait ses preuves à travers maints points de parenté surprenants avec les anciennes légendes des dieux et la mythologie allemande

9. Ibid., p. 282 / Ibid., p. 305 .

IO. J. et W. Grimm, KHM, I822, préface, p. Iv / Ibid., p. 9. 
a souvent eu l'occasion d'y revenir, elle a même trouvé dans la correspondance avec des mythes nordiques une preuve du contexte d'origine ${ }^{\text {II }}$.

La surprise que les Grimm mettent ici en avant, concernant les liens entre leurs Märchen et les cultures allemandes et nordiques, n'enlève rien à la scientificité affichée de leur recueil; au contraire, car ils affirment que la relation s'est établie en quelque sorte d'elle-même et qu'elle s'est présentée à eux sans qu'ils l'aient cherchée. Or, ce sont eux qui ont sélectionné (et rédigé) leur corpus, en effectuant des choix d'inclusion ou d'exclusion de tel ou tel récit; tout comme ce sont eux qui ont sélectionné les témoignages.

À ce sujet et en guise de transition au questionnement suivant, il convient de mentionner deux omissions dans les "Zeugnisse». La première, seulement partielle, concerne le refus de référence à un auteur de contes français comme Perrault, malgré la présence de la langue française dans les «Témoignages». L'omission de Perrault n'est pas complète, puisqu'il est en effet explicitement nommé par Eloi Johanneau dans le témoignage n ${ }^{\circ} 33$ :

On connaît aussi les contes de fées, du chat botté et du Petit Poucet avec ses bottes de sept lieues, contes populaires de la plus haute antiquité, qui ne sont point de l'invention de Perrault ${ }^{\mathrm{I} 2}$.

On le voit, cette mention nie la paternité des contes de Perrault, tout en mettant l'accent sur leur antiquité. Comme le diront les Grimm ensuite dans la section «Littérature», ces contes viennent de la «tradition orale» (mündlicher Überlieferung ${ }^{13}$ ). Dans la partie "Témoignages", ils cèdent la parole à autrui, en l'occurrence un francophone, pour préparer le terrain de leurs affirmations.

La deuxième omission est l'absence de toute citation en italien ou référence à la littérature italienne. Ce silence est d'autant plus frappant que dans les annotations ainsi que dans la section «Littérature», deux auteurs italiens, Straparola et Basile, reçoivent une attention particulière. Ce déséquilibre entre la partie "Témoignages» et «Littérature» est aussi perceptible si l'on examine la situation de l'espagnol : face aux trois citations dans les "Zeugnisse», l'Espagne ne se voit accorder que sept lignes dans la section "Littérature». Alors que presque dix pages de celle-ci traitent de Straparola et Basile, aucune citation en italien n’apparaît dans les «Témoignages». Une des raisons de cette absence peut résider dans le simple respect de l'économie du texte, de l'évitement des redites. Encore faut-il se demander la raison du

II. J. et W. Grimm, KHM, I837, préface, p. xxiv / H. Rölleke, KHM, 20Io, I, p. 25.

I2. J. et W. Grimm, KHM, I856, p. 28I / H. Rölleke, KHM, 20I0, III, p. 304.

I3. Ibid., p. $300 /$ Ibid., p. 324. 
placement d'un élément dans l'une ou l'autre section. Prenons un exemple concernant Straparola. Dans la partie «Littérature», les Grimm intègrent même une citation (en traduction allemande) dans leur présentation de l'auteur :

Straparola hat sie [die Märchen], wie es in der Vorrede zu dem zweiten Bande (vor der bten Nacht) heisst "aus dem Munde zehn junger Fräulein aufgenommen".

Straparola les [les contes] a, comme il est dit dans le prologue de la deuxième partie (avant la sixième Nuit), "reçus de la bouche de dix jeunes demoiselles » ${ }^{\mathrm{I}}$.

Ces affirmations reprennent celles de Straparola lui-même :

[...] holle [le piacevoli favole] fedelmente scritte secondo il modo che furono da dieci damigelle nel concistorio raccontate.

[...] je les [les fables plaisantes] ai écrites fidèlement selon la façon dont elles furent racontées par dix demoiselles dans l'assemblée ${ }^{15}$.

L'Italien met l'accent davantage sur l'activité narrative des jeunes femmes qui auraient raconté les histoires rapportées des Nuits facétieuses, ouvrage destiné, selon la même préface du livre II, à de "gracieuses et aimables femmes " (alle graziose e amorevoli donne $e^{16}$ ). Nous sommes donc apparemment face à des récits de jeunes femmes (et non de vieilles femmes) racontés pour des femmes (et non pour des enfants) : cela ne correspond pas à la scénographie des Märchen que les Grimm établissent, dès le deuxième volume publié en I8I5, par le biais de la narratrice paysanne appelée la "Viehmännin» (voir à ce sujet la note I4 de la contribution de U. Heidmann). D'où peut-être le silence des "Zeugnisse» sur Straparola.

Une chose semblable paraît se passer dans le cas de Basile. Là non plus, le cadre du récit n'est pas le foyer familial, mais une tente plantée dans un jardin princier; nous y trouvons surtout des narratrices du peuple, mais n'y rencontrons toujours pas de femmes âgées ou de mères et le public n'est pas enfantin. Le prince, il est vrai, s'adresse à un cercle de narratrices auxquelles il a demandé de «raconter journée après journée chacune un conte, du type de ceux que les vieilles racontent d'ordinaire pour divertir les petits enfants" (de contare ogni iornata no cunto ped uno, de chille appunto che soleno dire le vecchie pe trattenemiento de peccerille $\left.{ }^{\mathrm{17}}\right)$. Les Grimm ne citent pas ce passage,

I4. Ibid., p. 286 / Ibid., p. 309.

I5. Straparola, Le piacevoli notti, lettre dédicatoire au début du deuxième livre; édition de

D. Pirovano, 2000, II, p. 425.

I6. Ibid.

17. Basile, Lo cunto de li cunti, Avertissement; édition de M. Rak, 1999, p. 22; traduction de

F. Decroisette, 2002, p. 37-38. 
car la comparaison marque davantage le décalage, du fait que les narratrices ne sont précisément pas des vieilles et que leur public n'est pas constitué d'enfants. Tout se passe «à la manière» de cette scénographie, mais sans y correspondre exactement. Malgré la fierté des Grimm d'avoir rendu sinon accessible du moins plus familier le texte de Basile ${ }^{\mathrm{I}}$, sa place ne peut pas être parmi les "Témoignages", car il ne correspond pas à la scénographie que les Grimm fabriquent pour leurs Märchen.

\section{Les citations des "Zeugnisse" comme moyen de créer la scénographie des Märchen}

Je n'entrerai pas ici dans les détails de la scénographie des KHM étudiée de façon bien plus approfondie par U. Heidmann, mais j'aimerais quand même en aborder certains aspects qui concernent les "Zeugnisse». Il sera en particulier question d'éléments qui relèvent de la mise en scène de l'acte de narration des contes - celui/celle qui raconte/écrit et celui/celle qui écoute/ lit - ainsi que du contexte (situation, lieu) dans lequel cet échange se déroule. Dans le cas des KHM, cette mise en scène est relativement complexe : si les auteurs en sont les Grimm, ces derniers affirment avoir collecté leurs matériaux à partir de sources orales ou écrites. Alors que, dans le titre, ces éléments sont inclus sous une forme très compacte, ils sont présentés plus en détail dans les "Zeugnisse». Je propose de passer en revue certains de ces éléments dans l'ordre des mots du titre Kinder-und Hausmärchen, gesammelt durch die Brüder Grimm ${ }^{19}$.

En ce qui concerne le premier mot, "Kinder(-märchen)», on constate que la figure de l'enfant est très présente dans les "Témoignages ${ }^{20}$. $\mathrm{Ne}$ pouvant pas m'attarder sur toutes les mentions d'enfants ${ }^{21}$, je me limiterai à deux aspects. D'une part, on voit que le personnage enfantin est mis en scène comme auditeur de contes, soit directement, soit dans les souvenirs évoqués par un adulte. D'autre part, une importance particulière est

I8. J. et W. Grimm, KHM, I822, préface, p. IV / H. Rölleke, KHM, 2oIo, III, p. Io : «Le mérite lui revient [à la partie "Littérature»] d'éveiller l'intérêt pour le Pentamerone de Basile et de le faire connaître dans tout son contenu, alors que d'ordinaire il est tout au plus mentionné selon son titre.» (Das Verdienst bleibt ihm [dem Abschnitt Literatur] den Pentamerone des Basile, der sonst höchstens dem Titel nach ist aufgeführt worden, näher und seinem ganzen Inhalte nach bekannt zu machen.)

19. Pour une analyse détaillée du titre en tant que «scénographie», voir la contribution de U. Heidmann.

20. D'ailleurs, si dans l'édition de I856, les témoignages sont présentés sous le titre de "Zeugnisse», ils étaient désignés dans la première édition de I8I2 comme des «Témoignages pour des contes pour enfants» (Zeugnisse für Kindermärchen) [J. et W. Grimm, KHM, I8I2, p. XXII].

2I. Les enfants sont mentionnés, voire mis en scène dans les témoignages suivants : ${ }^{0 s} 2,3,6,9$, II, I3, I5, I7, I8b, 2I, 22, 24, 26, 28, 30, 3I. 
accordée aux effets sur ce public. Une facette de la réception me semble particulièrement intéressante : la présence de la peur suscitée par des contes qui sont loin d'être toujours plaisants. Dès les premiers "Témoignages", il est question de figures monstrueuses, comme la Gorgone $\left(\mathrm{n}^{\circ} 2\right)$ ou la Lamie $\left(\mathrm{n}^{\text {os }} 2\right.$ et 6$)$. Dans le deuxième témoignage de Strabon, ces contes, selon l'ajout en allemand des Grimm, sont "horribles» (schrecklich) et servent à "prévenir» (abzuhalten) les enfants de mauvais comportements ${ }^{22}$. Dans ce sens, les monstres sont mis en relation avec le but éducatif des contes. Cela se perçoit aussi dans un témoignage beaucoup plus tardif, le $\mathrm{n}^{\circ} \mathrm{I} 5 \mathrm{de}$ Joachim Camerarius, datant du XVI ${ }^{\mathrm{e}}$ siècle, où il est question de récits dont "l'exposition était tournée en un certain mode effrayant» (expositionem ad abhorrentem quendam modum deflexam ${ }^{23}$ ). Là-dessus, le témoignage continue en parlant de la terreur (terrore), de la peur (formidine), de l'espoir (spe), de la joie (laetitia), que les récits devraient insuffler au peuple (vulgi) et aux enfants (puerorum) en vue de les amener à une certaine religion (religione quadam $)^{24}$.

L'entrée en jeu de la religion n'est pas anodine si l'on sait qu'en allemand, le mot Mare, d'où vient le diminutif Märchen, est en lien avec la "Bonne Nouvelle»; comme on le voit dans la fameuse chanson de Noël de I539 de Luther où l'on annonce la "gute neue $M a ̈ h r^{25}$ ». En outre, deux des témoignages des Grimm concernent précisément Luther. Le n ${ }^{\circ}$ i3 le cite (sans donner la référence exacte), disant qu'il n'aimerait «pour tout l'or du monde» (um kein Gold) se défaire des «merveilleuses histoires» (wundersamen Historien) de sa "tendre enfance» (aus zarter Kindheit ${ }^{26}$ ). Dans le deuxième ( $\left.\mathrm{n}^{\circ} 22\right)$, Gregor Balthasar Schuppius parle longuement de l'intérêt de Luther pour les contes et de son désir d'en écrire. Nous avons donc, en plus de l'effroi causé par certains contes, l'établissement d'un lien avec l'éducation et la religion. Ceci était encore plus marqué dans la première version des "Zeugnisse», qui comptait six témoignages, dont précisément celui de Strabon ( $\left.\mathrm{n}^{\circ} 2\right)$ et deux de Luther ( $\mathrm{n}^{\text {os }}$ I3 et 22).

Tout en restant importante, cette relation s'atténue ou se modifie dans la suite des "Zeugnisse» dont le chiffre augmente à 37 et dans lesquels on découvre des témoignages se référant à des contes d'effroi en quelque sorte, sans les mettre explicitement en lien avec une valeur éducative, mais en

22. J. et W. Grimm, KHM, I856, p. 273 / H. Rölleke, KHM, 20I0, III, p. 297.

23. Ibid., p. 275 / Ibid., p. 299.

24. Ibid., p. $275-276$.

25. H. Rölleke, Die Märchen der Brüder Grimm, 2010, p. Iо.

26. J. et W. Grimm, KHM, I856, p. 275 / H. Rölleke, KHM, 2010, III, p. 299. 
mettant l'accent sur le plaisir créé par de tels récits. Dans le ${ }^{\circ} 26$, attribué à un certain Rabener, on lit par exemple :

Liebe Amme, ich erinnere mich der langen Abende noch immer mit Vergnügen, an denen ich als ein junger Knabe auf deinem Schoße saß und meinen zitternden Arm ängstlich um deinen Hals schlang, wenn du uns das fürchterliche Märchen vom Seehunde, das traurige Märchen vom verwünschten Prinzen ohne Kopf, oder das fromme Märchen vom lahmen Esel erzähltest [...]

Chère nourrice, je me souviens encore toujours avec plaisir des longues soirées pendant lesquelles, jeune garçon, j'étais assis sur tes genoux et, empli d'effroi, j'enlaçais ton cou de mon bras tremblant, quand tu nous racontais le terrible conte du phoque, le triste conte du maudit prince sans tête, ou le conte pieu de l'âne boiteux $[\ldots]^{27}$

L'effroi se combine avec la fascination des enfants pour le sujet, décrite dans le portrait rapporté dans le témoignage $\mathrm{n}^{\circ}$ 3I. Il s'agit d'une citation que les Grimm tirent de la traduction allemande, par August von Rode, du texte anglais The Pleasures of Imagination de Marc Akenside. Le passage est tellement suggestif que je ne peux m'empêcher de le citer :

Darum fesselt

Das Mütterchen zu nacht beim hellen Herd

Der Kinder willig Ohr durch schauervolle

Geschichtchen von Bezauberungen und

Von bösen Geistern, von der Todtenuhr,

Die fürchterlich dem Bösewichte tönet,

Durch die die Witwe darbt, die Waise weint:

Von Seelen, die, geheime Schuld zu mildern,

Rastlos dem Grab entstiegen: von Gespenstern,

Die Ketten schleppend, Höllenbrände schwingend,

Um Mitternacht des Mörders Bett umspuken.

Bei jeder Pause weichen schüchtern alle

Zurück und sehn sich an: mit kalter Angst

Durchrieseln Schauer sie; doch nach dem Ausgang

Begierig, dringen bald zum Mütterchen

Sie wieder hin, vor süßer Furcht kaum athmend.

C'est pourquoi, suspendus aux lèvres

De la petite mère assise la nuit près du foyer,

Les enfants, volontiers captivés par de terrifiantes

Petites histoires d'enchantements et

De mauvais esprits, de l'horloge des morts,

Qui résonne terriblement aux oreilles du méchant,

[Horloge] à travers laquelle la veuve est dans le besoin, l'orpheline pleure :

[Histoires] d'âmes qui, pour apaiser une faute secrète,

27. Ibid., p. 279 / Ibid., p. 302. 
Sans repos montent de la tombe; [histoires de fantômes],

Qui, traînant des chaînes, agitant des feux infernaux,

À minuit, hantent les abords du lit du meurtrier.

À chaque pause, tous reculent timidement

Et se regardent : ruissellent en eux

Des frissons de peur froide;

Mais désireux du dénouement,

Ils se rapprochent de nouveau de la petite mère,

De nouveau, ne respirant presque pas, dans un état de douce peur ${ }^{28}$.

À côté de l'idée d'effroi, on trouve aussi celles de rétribution et de punition, donc de but éducatif des contes. Mais la nature du plaisir des enfants ici décrit invite à la réflexion, car nombre de Märchen des Grimm sont loin de présenter un monde idyllique dépourvu de violence. Au contraire, plusieurs récits rapportent des histoires sanglantes. Cet aspect de leurs contes leur a valu plusieurs critiques et on sait qu'ils ont, avec le temps, enlevé ou atténué certains éléments violents ${ }^{29}$. Dans la préface de I8I9, ils affirment par exemple :

Dabei haben wir jeden für das Kinderalter nicht passenden Ausdruck in dieser neuen Auflage sorgfältig gelöscht.

Dans ce sens, nous avons soigneusement effacé dans cette nouvelle édition toute expression inadaptée au jeune âge ${ }^{30}$.

Et, dans la préface de 1837, ils expliquent une des raisons d'être de la «Petite édition" (Kleine Ausgabe) de leurs contes :

Eine Auswahl, als kleinere Ausgabe in einem Bändchen, wobei zugleich die Bedenklichkeit derer berücksichtigt ist, welche nicht jedes Stück der grösseren Sammlung für Kinder angemessen halten, veranstalteten wir zuerst I825, sie ist 1833 und I836 wieder aufgelegt worden.

Cette sélection, sous forme d'édition mineure en un petit volume, pour prendre en compte l'inquiétude de ceux qui estiment que les pièces de la collection majeure ne sont pas toutes adaptées à des enfants, nous l'avons préparée d'abord en I825; elle a été rééditée en 1833 et $1836^{31}$.

28. Ibid., p. 279-280 / Ibid., p. 303. Il s'agit du seul texte anglais traduit en allemand des "Témoignages"; or le traducteur n'est autre que August von Rode, le plus fameux traducteur d'Apulée, dont la traduction isolée du récit de Cupidon et Psyché, suivie de celle de tout le texte d'Apulée fait encore aujourd'hui référence. Aurions-nous là un clin d'œil à Apulée qui pourtant n'a presque pas de place dans les «Zeugnisse»?

29. Voir par exemple N. Rimasson-Fertin, Contes pour les enfants et la maison, 2009, II, p. 584-590. Pour une étude plus pointue des contes d'effroi dans les KHM, voir le chapitre "Contes d'effroi et contes de mise en garde", dans J.-M. Adam et U. Heidmann, Le texte littéraire, 2009, p. 4I-45.

30. J. et W. Grimm, KHM, I819, préface, p. viII / H. Rölleke, KHM, 20Io, I, p. I7.

3I. J. et W. Grimm, KHM, i837, préface, p. XxiII-XXIV / Ibid., p. 24-25. 
Malgré tout cela, la violence, l'effroi, les crimes sanglants sont présents dans les KHM. L'inclusion des Zeugnisse que nous venons d'évoquer sert à légitimer cette présence de l'horrible et de l'effroi dans les contes ${ }^{32}$.

Les deux derniers témoignages cités ( $\mathrm{n}^{\text {os }} 26$ et 3I) introduisent une figure absente du titre des KHM, mais primordiale pour la scénographie des Märchen : celle du narrateur de contes ou, plus précisément, de la narratrice. Dans le $\mathrm{n}^{\circ}$ 26, il était question d'une «nourrice» (Amme) et, dans le $\mathrm{n}^{\circ}$ 3I, d'une "petite mère» (Mütterchen). Ce ne sont de loin pas les seules figures féminines qui racontent des contes dans les "Zeugnisse». À côté du personnage enfantin, c'est la figure de la femme qui apparaît le plus souvent et dans la position de productrice du récit. Si l'on rencontre des fileuses ou des vieilles femmes, les figures maternelles de la mère ou de la nourrice sont les principales narratrices de contes ${ }^{33}$. Par cette présence de la femme narratrice et plus spécifiquement de la femme maternelle narratrice, les Grimm légitiment deux caractéristiques de la scénographie de leur œuvre : d'une part, leurs propres femmes conteuses qu'ils mettent ici en scène (tout en les taisant dans leurs contes ${ }^{34}$ ); de l'autre, le tableau familial que ces figures maternelles servent à dresser.

Ceci nous conduit au deuxième mot du titre des KHM, «Hausmärchen», moins mis en avant dans les "Témoignages». Il est par exemple question de raconter "auprès du feu, avec sa famille, tard le soir" (apud ignem cum familia sua de sero $^{35}$ ), des récits "avec lesquels on se distrait auprès du feu pendant les longues nuits d'hiver" (con que se entretienen al fuego las dilatadas noches del invierno ${ }^{30}$ ), ou encore de "longues soirées d'hiver dans une salle de séjour sans lumière» (bei langen Winterabenden in einer Stube ohne Licht ${ }^{37}$ ).

32. Dans ce sens, il est intéressant de constater que le dernier témoignage, le $\mathrm{n}^{\circ} 37$, n'est pas simplement une recension des KHM : les Grimm l'introduisent en disant que l'article parle du conte du genévrier (Märchen vom Machandelboom), un des contes les plus sanglants, qui a perduré dans le recueil depuis ses débuts.

33. Pour la statistique : face à un seul grand-père ( $\left.\mathrm{n}^{\circ} \mathrm{I} 8\right)$, on a des fileuses $\left(\mathrm{n}^{\mathrm{os}} \mathrm{I} 4,17,23\right)$, des vieilles femmes $\left(\mathrm{n}^{\mathrm{os}} 5,7,16,24,37\right)$ et surtout des mères ou des nourrices $\left(\mathrm{n}^{\mathrm{os}} 3,4,6,20,26,30,31,32,34\right.$, 36).

34. Encore faut-il ajouter, comme le rappelle J. E. Sennewald, que dans les éditions postérieures, les exemples de mises en scène d'une narration (implicitement de conte) par une figure maternelle augmentent; l'exemple qu'il donne est un passage du Schneeweißchen und Rosenrot (Das Buch, das wir sind, 2004, p. 3I n. 42).

35. J. et W. Grimm, KHM, I856, p. 274 / H. Rölleke, KHM, 20IO, III, p. 298.

36. Ibid., p. 276 / Ibid., p. 299.

37. Ibid., p. 279 / Ibid., p. 302. 
Il nous reste maintenant à considérer les «Zeugnisse» à partir de la dernière partie du titre des KHM, titre qu'il convient d'avoir toujours en tête sous sa forme étendue, car, comme le montre l'analyse de U. Heidmann et comme l'indique J. E. Sennewald : "[...] la mention du mode ("collectés") et celle des responsables du livre ("par les frères Grimm") sont partie constituante du titre.» ([...] die Nennung des Modus ("gesammelt") und der Verantwortlichen für das Buch ("durch die Brüder Grimm") [sind] Bestandteil des Titels $\left.3^{8}\right)$. La première intuition serait de penser que cet aspect primordial de l'œuvre des Grimm ne devrait pas forcément être reflété dans les "Témoignages", qui portent plus sur les Märchen que sur leur collecte. Faut-il corriger cette impression? Si l'on y regarde de près, un passage semble fort intéressant à ce sujet; il s'agit d'une citation du Jucundi Jucundissimi de Johann Beer donnée au témoignage $\mathrm{n}^{\circ} 23$ :

Dergestalt kamen wir an diesem Ort zusammen, alwo das Gesind das Werg abzuspinnen pflegte. Weil es nun ohnedem der Gebrauch war daß einer nach dem andern ein Märlein oder andere Geschicht erzählen musten. Dann die Wahrheit zu gestehen, so hatte an solchen Erzählungen nicht allein die Edelfrauen sondern auch ich und der Schreiber unser ganzes Vergnügen, gestaltsam wir dann oftermalen die alten Bettler zu solchen Erzählungen angehalten und ihnen um einen Zweier mehr spendiert.

C'est ainsi que nous nous réunissions à cet endroit où les domestiques avaient l'habitude de filer l'étoupe; puisque c'était de toute façon l'usage de raconter l'un après l'autre un conte ou une autre histoire. Et puis, à vrai dire, non seulement les femmes nobles, mais moi aussi et le scribe, nous prenions pleinement plaisir à ce genre de récits, de sorte qu'alors nous invitions souvent de vieux mendiants à raconter ce genre de récits et que nous leur offrions de surcroît environ deux sous ${ }^{39}$.

Comme dans une sorte de tableau, nous nous trouvons en présence de figures de collecteurs de contes qui ressemblent à ceux de la scénographie des KHM, sans que les Grimm n'ajoutent aucun commentaire. Le témoignage est au discours direct, présentant et légitimant ainsi l'activité de collecteurs semblable à celle que les Grimm s'octroient. Or, comme ils ne sont jamais allés collecter les contes, il est intéressant de constater que ce portrait reste en quelque sorte à distance, et cela doublement. D'une part, le texte cité, le Jucundus Jucundissimus, est loin d'être un ouvrage qui reflète uniquement la réalité. Les mots du titre suffisent à montrer qu'on entre dans un univers différent, car il est question d'un "récit de vie curieux" (wunderliche Lebensbeschreibung), de "maints événements aventureux et bizarres» (lauter

38. J. E. Sennewald, ouvr. cité, p. I3.

39. J. et W. Grimm, KHM, I856, p. 278 / H. Rölleke, KHM, 20IO, III, p. 30 I. 
abentheurliche und seltsame Begebenheiten $\left.{ }^{40}\right)$. D'autre part, nous avons relevé l'absence de remarque ou d'intrusion quelconque de la part des Grimm dans le passage. En soi, ce manque n'est pas surprenant, puisque, dans la section des "Témoignages», il s'agit précisément de laisser parler d'autres personnes. Mais devraient-ils pour autant se taire complètement?

\section{La citation de témoignages comme moyen de se donner une autre voix}

À lire la préface de I822 au troisième volume des KHM, il semblerait que ce silence soit volontaire, puisque les Grimm y parlent des Zeugnisse en disant :

[...] sie enthalten Urtheile über ihren Werth [der Märchen], die um so mehr Gewicht haben als sie ohne Vorliebe, parteilos und gelegentlich, von Männern sind geäußert worden, welche sich einen freien und unbefangenen Blick bewahrt hatten.

[...] ils contiennent des jugements sur leur valeur [des contes] qui n'ont que plus de poids puisqu'ils ont été exprimés sans préférence, de façon impartiale et occasionnellement par des hommes qui avaient conservé un regard libre et sans préjugétr.

Cette citation est intéressante à plusieurs égards. Je propose de l'aborder en trois étapes, en commençant par la fin. Il convient en effet d'attirer l'attention sur le temps verbal employé, le plus-que-parfait : «ils avaient conservé» (sich [...] bewahrt hatten). Par ce choix de l'accompli, les auteurs des témoignages évoqués sont placés temporellement à distance. Or, si cet éloignement temporel est particulièrement grand pour les premiers témoignages provenant de l'Antiquité, les autres témoignages, comme je l'ai déjà dit, se rapprochent de plus en plus des Grimm, jusqu'aux derniers qui leur sont contemporains. Ces derniers sont cependant placés par la préface à une même distance des KHM que les autres Zeugnisse et cela en dépit du fait que c'est précisément la publication de l'œuvre des Grimm qui les a motivés, puisqu'il s'agit de réactions ou de recensions des KHM. De plus, ce sont des échos positifs auxquels leur emplacement, dans la continuation d'une chaîne bien plus ancienne, confère une aura d'indépendance par rapport aux KHM et de ce fait une apparente impartialité.

Le deuxième point que j'aimerais relever dans la citation de la préface est celui de l'objectivité attribuée aux auteurs des Zeugnisse, qui auraient écrit "sans préférence, de façon impartiale" (ohne Vorliebe, parteilos) et avec «un regard libre et sans préjugé» (freien und unbefangenen Blick). La plupart des exemples que nous avons examinés ne sont cependant pas

40. J. Beer, Jucundi Jucundissimi, I680, page de titre.

4I. J. et W. Grimm, KHM, I822, préface, p. IV / H. Rölleke, KHM, 20ı, III, p. 9-IO. 
tout à fait neutres : il y est question des plaisirs suscités par les contes et des souvenirs agréables qui leur sont rattachés. À côté de ces exemples, qui ne sont pas des exceptions dans la liste des témoignages, il y a certes des cas plus détachés, notamment par l'absence de relation personnelle au conte, mais là aussi, les jugements sont tous positifs. Cependant, l'absence de sentimentalisme dans certains témoignages invite à leur attribuer une certaine neutralité, effet qui s'étend aux autres témoignages, si l'on se laisse guider par les indications de la préface.

Le troisième point qui mériterait d'être discuté bien plus longuement que je ne peux le faire ici concerne l'autorité des témoignages, attribuée à d'autres personnes que les Grimm («exprimés [...] par des hommes» [von Männern sind geäußert worden]). S’il est vrai que certains des Zeugnisse sont cités tels quels, avec uniquement la mention de l'auteur, suivie éventuellement par une référence plus ou moins exacte, ce n'est pas le cas pour tous les témoignages. On trouve en effet plusieurs fois des commentaires des Grimm. Ces indications peuvent précéder la citation $\left(\mathrm{n}^{\circ} 37\right)$ ou la suivre $\left(n^{\circ} 6\right)$, voire les deux ( $\left.n^{\circ} 9\right)$. Les remarques des Grimm peuvent même presque s'enchevêtrer avec la citation, comme c'est le cas au $\mathrm{n}^{\circ} 24$, placé sous l'autorité d'Ernst Joachim von Westphalen :

Narrant [mulierculae] multo verborum apparatu historiolas vom Blocksberg, von der schwarzen Hexe, von dem Kerl der die Kinder in den Sack steckt, vom Dühmling, vom König Blaubart, von der Kukukssuppe, vom Drutenfuß, der alten Eten Inne, von der Königstochter im blauen Thurm, et infinitas fabulas.

Elles [les petites femmes] racontent avec un langage très pompeux les petites histoires $\mathrm{du}$ Blocksberg, de la sorcière noire, du gars qui fourre les enfants dans le sac, du Dühmling, du roi Barbe bleue, de la soupe du coucou, du Drutenfuß, de la vieille Eten Inne, de la fille du roi dans la tour bleue, et des fables sans fin ${ }^{42}$.

La citation qui commence en latin est interrompue par un ajout en allemand avec des titres de contes pour finir sur le latin. À la fin de la citation suivent d'autres remarques du même type apportées en allemand par les Grimm. Or, si dans ce cas, en raison de l'ajout final, on penche pour interpréter les parties en allemand comme étant le discours des Grimm, ce n’est pas toujours aussi évident. Voici comme se présente le témoignage $\mathrm{n}^{\circ} 30$ de Johannes Müller :

42. J. et W. Grimm, KHM, I856, p. 278 / Ibid., p. 302. Dans la traduction, je garde en allemand (indiqué en italique) les expressions enfantines ou dialectales se référant à des sortes de titres de contes. 
Man sollte die Weisheit der Völker, bei denen man lebt, in ihrer mannigfaltigen

Gestalt, selbst in Liedern,

quas ad ignem aniculae

narrant puellis,

aufspüren und in Umlauf bringen.

On devrait chercher et faire circuler la sagesse des peuples, auprès desquels on vit, dans sa forme variée, même dans les chansons,

qu'auprès du feu les vieilles

racontent aux jeunes filles ${ }^{43}$.

Pour la partie en latin, on suppose qu'il s'agit d'une citation, mais pour les paroles encadrantes en allemand, on peut se demander de qui elles émanent : des Grimm ou de leur source Johannes Müller? En fait, on peut parler d'une sorte de mélange de voix à travers lequel la voix des Grimm est amalgamée, d'une certaine façon, avec celle de leur source. La situation est ici en effet la suivante : le texte apparaît presque tel quel dans la source indiquée, avec l'allemand encadrant le latin, mais les Grimm ont légèrement modifié la partie allemande. Le texte cité est tiré d'une recension traitant d'un recueil de dictons populaires couplés à des fables et Müller, l'auteur du compte rendu, affirme élogieusement souhaiter que l'auteur du recueil, "Monsieur Ferrich continue à chercher et à faire circuler la sagesse des peuples auprès desquels il vit" (dass Herr Ferrich [...] fortfahre, die Weisheit der Völker, bei denen er lebt [...] aufzuspüren, und in Umlauf zu bringen $\left.{ }^{44}\right)$. D'une affirmation bien concrète, appliquée à une personne en particulier, Herr Ferrich, nous passons, avec les Grimm, à une version généralisée, avec un "on» (man) applicable à tout le monde. Cela est fait par une voix qui, à la simple lecture et si l'on ne va pas vérifier la source, mêle les Grimm et Müller. Or, si l'on s'en tient à l'original, on voit bien que les Grimm ont légèrement reformulé le discours de Müller selon leurs propres buts.

Dans d'autres cas, les Grimm substituent même leur propre discours à celui des auteurs de leurs témoignages : ils renoncent à la citation en faveur de la paraphrase, voire à la simple mention résumée de ce que dit la source, pour ne retenir que ce qui les intéresse ${ }^{45}$. Un exemple de ce dernier procédé se trouve dans le témoignage $\mathrm{n}^{\circ} 25$, qui consiste en une seule phrase : «Des "petits contes" et "petites histoires" lui ont été racontés pendant son enfance» (Ihm werden in seiner Kindheit "Mährlein und Histörchen" erzählt"6).

43. Ibid., p. 279 / Ibid., p. 303.

44. J. von Müller, Sämmtliche Werke, I8II, p. 245.

45. Autres exemples de la mention de témoignages en paraphrase $: n^{\text {os }} 3,18,27$.

46. J. et W. Grimm, KHM, I856, p. 278 / H. Rölleke, KHM, 2010, III, p. 302. 
On trouve aussi des cas où la fluctuation et la substitution de voix se combinent, au point qu'on pourrait parler d'une sorte d'appropriation non déclarée. Il est vrai que les Grimm fournissent, avant de présenter un témoignage, une référence plus ou moins exacte du passage. Malgré cela, il arrive qu'ils citent sans le laisser clairement percevoir. C'est notamment le cas des témoignages en langues étrangères. Prenons comme exemple le $\mathrm{n}^{\circ} 2$ sur Strabon. Les Grimm donnent le texte suivant :

Wir erzäblen den Kindern liebliche Märchen zur Ermunterung (

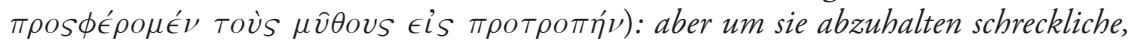
wie jene von der Lamia, der Gorgone, von Ephialtes und Mormolyk.

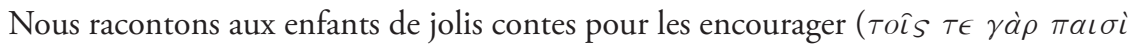

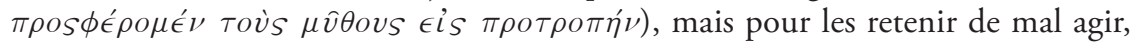
[nous leur racontons des contes] horribles, comme ceux de la Lamie, de la Gorgone, d'Éphialte et Mormolycée ${ }^{47}$.

Si la première partie de la citation est présentée, par l'ajout du grec entre parenthèses, comme une traduction du texte de Strabon, cela ne semble pas être le cas pour la deuxième moitié du passage. Comme elle est entièrement en allemand, on aura tendance à la rattacher au discours des Grimm. Or, si l'on s'attarde sur l'original, on constate que le témoignage dans son entier s'avère être une traduction. Voici le passage tel qu'il se présente chez Strabon :

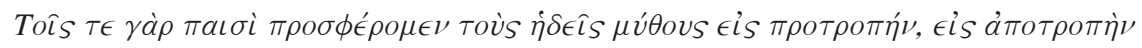

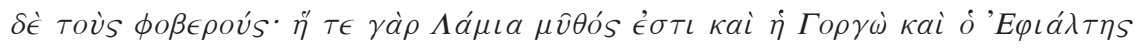

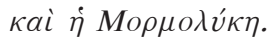

En effet, nous présentons aux enfants des mythes agréables pour les encourager, effrayants pour les dissuader; en effet, Lamie est un <tel> mythe, ainsi que Gorgone et Éphialte et Mormolycé ${ }^{4}$.

La traduction s'est donc d'une certaine façon transformée en discours des Grimm eux-mêmes qui ont repris les paroles de Strabon en l'indiquant seulement en partie. Par ailleurs, ils ont même retranché le texte de la source grecque, puisque, dans leur version, l'adjectif «agréable» ( $\dot{\eta} \delta \in \hat{\imath} s)$ de l'original n’apparaît pas en grec, mais seulement en allemand, «joli»(lieblich). Si j'insiste sur ces exemples qui peuvent sembler accessoires, c'est parce que ces détails révèlent la façon subtile des Grimm de s'approprier le discours d'autrui pour se donner une autre voix qui les légitime, non seulement eux, mais aussi les Märchen de leur ouvrage. Les façons de faire que nous

47. Ibid., p. 273/ Ibid., p. 297; l'erreur dans le grec (le sigma final à l'intérieur du mot « $\pi \rho \circ \phi \phi \epsilon ́ \rho o \mu \epsilon \nu$ » au lieu de " $\pi \rho \circ \sigma \phi \epsilon ́ \rho \rho \epsilon \nu »)$ se trouve dans la citation des Grimm.

48. Strabon, I, 2, 8; édition de A. Meineke, I969. 
venons d'évoquer ne se limitent certainement pas aux «Témoignages»; elles sont probablement mises en pratique dans l'écriture des contes.

Un dernier élément qu'il convient de mentionner ici est le choix non seulement des œuvres citées mais aussi et surtout des passages sélectionnés par les Grimm et, ce qui va ensemble, des extraits omis. Je n'évoquerai que deux exemples. Le premier concerne Apulée. Les travaux de U. Heidmann ont mis en avant l'importance pour les contes de l'histoire de Cupidon et Psyché enchâssée dans les Métamorphoses ${ }^{49}$. Les Grimm se réferent à ce récit, en le désignant comme "Legende», mais surtout en le qualifiant de "Märchen " ${ }^{\circ}$. Dans les "Témoignages", on ne trouve qu'une seule et très brève citation d'Apulée, dans le $\mathrm{n}^{\circ} 5$ : «Mais moi, je vais te distraire tout de suite avec des récits agréables et des fables de vieille» (Sed ego te narrationibus lepidis anilibusque fabulis protinus evocabo ${ }^{\text {sI }}$ ). Et pourtant, dans une des remarques notées à la main sur les pages de la première édition de I8I2 contenant les "Zeugnisse», les Grimm écrivent clairement : "Apulée. Conte d'Amour et Psyché» (Apulejus. Märchen von Amor und Psychés2). Malgré cela, ils n'ajoutent aucune autre citation dans les éditions ultérieures des «Témoignages» et, surtout, ils n'y incluent pas la clôture de l'histoire de Cupidon et Psyché :

Sic captivae puellae delira et temulenta illa narrabat anicula, sed astans ego non procul dolebam mehercules quod pugillares et stilum non habebam qui tam bellam fabellam praenotarem.

49. Pour l'importance de la fabella de Cupidon et Psyché pour le conte, je me permets de renvoyer aux études de U. Heidmann, dont je mentionnerais ici en particulier : «La Barbe bleue palimpseste», 2008; "Comment faire un conte moderne avec un conte ancien?", 2009; "La (re)configuration des genres dans les littératures européennes", 2009; "Enjeux d'une comparaison différentielle et discursive", 20I0; "Intertextualité et dialogicité des contes", 20I0; "Perrault en dialogue avec Apulée, Fénelon et Lhéritier», 20IO; "Quel apport du comparatisme pour l'étude des cultures", 20I0; "Expérimentation générique et dialogisme intertextuel », 20II; "Tisserandes fatales (Apulée) et fées de cours (Perrault)", 20II.

50. Dans la partie de la section sur la littérature portant sur d'Aulnoy, les Grimm se réfèrent d'abord à la "légende d'Amour et Psyché» (Sage von Amor und Psyché [J. et W. Grimm, KHM, 1856, p. 304 / H. Rölleke, KHM, 20IO, III, p. 329]), puis au "conte d'Amour et Psyché» (Märchen von Amor und Psyché [Ibid., p. 305 / Ibid.]). Dans l'annotation du conte $\mathrm{n}^{\circ} 88$, "Das singende springende Löweneckerchen", il y a tout un développement sur le "si fameux conte de Psyché» (so bekannte Märchen von der Psyche [Ibid., p. I55 / Ibid., p. 173]).

5I. Ibid., p. 273 / Ibid., p. 297. Lerreur dans la citation latine, «evocabo" au lieu de «avocabo», est dans la citation des Grimm.

52. J. et W. Grimm, KHM, I8ı2, p. XxiII. 
Ainsi, à la jeune captive la vieille délirante et ivre racontait ces choses, mais moi, me tenant debout à côté, j'étais affligé, par Hercule, de n'avoir ni tablettes ni stylet avec lesquels j'aurais pu noter un si beau petit récit ${ }^{53}$.

On sait que le narrateur principal des Métamorphoses est le jeune Lucius qui raconte ses aventures, avant, pendant et après sa transformation en âne. Ce narrateur cède la parole à plusieurs personnages, dont une vieille femme qui raconte l'histoire de Cupidon et Psyché. Par ces affirmations que Lucius place à la fin de ce récit, ce dernier reçoit une tout autre signification. Comme le note U. Heidmann, cette remarque finale «explicite le fait que la fabella de Psyché que nous venons de lire n'est pas ce que la vieille a raconté, mais le récit que le narrateur Lucius en a fait ultérieurement, après avoir retrouvé sa forme humaine et la capacité de tenir un stylet et une tablette ${ }^{54}{ }^{\prime}$. C'est peut-être précisément à cause de cette explicitation selon laquelle ce n'est pas la vieille qui a raconté l'histoire, que ce passage n'a pas été inclus par les Grimm dans leurs témoignages. De plus, le portrait d'une vieille «délirante et ivre» (delira et temulenta) n'aurait pas été conforme à l'image de la narratrice de contes telle qu'ils la construisent dès le deuxième volume publié en I8I5. On peut donc parler d'une omission par protection de leur propre projet générique.

Dans l'exemple suivant, le ${ }^{\circ}$ I4 avec une citation de La perfecta casada de Fray Luis de León, on est plutôt confronté à une lacune par désintérêt pour la partie délaissée. Pour le dire autrement, est omis de l'extrait ce qui ne soutient pas les buts poursuivis. Les Grimm citent ainsi la source espagnole :

$Y$ verá que estándose sentada con sus mugeres volteando el huso de la mano y contando consejas — se texe la tela y se labra el paño.

Et vous verrez qu'étant assise avec ses femmes, la main tournant le fuseau, et tout en racontant des récits moraux — on tisse la toile et on travaille le drap ${ }^{55}$.

Le tiret signale, dans cette citation, l'omission d'un passage dans lequel la femme dont il est ici question, parfaite épouse dont l'ouvrage veut faire le portrait, est longuement comparée à un navire plein de richesses. Cela n'intéresse pas les Grimm, d'où cet effacement. Mais, dans le chapitre 6 de La perfecta casada d'où est tirée la citation, la comparaison avec le riche navire est le sujet principal. En revanche, dans le chapitre 5, il était très longuement question précisément de filage, de tissage dans une longue liste de fileuses allant de Pénélope à la reine Isabelle la Catholique. Aucun de ces portraits ne retient l'attention des Grimm, qui leur préfèrent le passage

53. Apulée, Métamorphoses VI,25,I; texte établi par D. S. Robertson, 2002.

54. U. Heidmann, «Intertextualité et dialogicité des contes», 20I0, p. 56.

55. J. et W. Grimm, KHM, I856, p. 275 / H. Rölleke, KHM, 20I0, III, p. 299. 
cité. La raison en est que, dans l'exemple choisi, Luis de León mentionne non seulement le tissage, mais aussi la narration, élément qui n'est pas mis en avant dans les tableaux du chapitre 5, alors que c'est précisément ce qui intéresse les Grimm. Par ailleurs, et c'est important pour notre propos comparatiste, ils trouvent, dans la citation sélectionnée, le terme espagnol qu'ils vont choisir comme traduction de Märchen : conseja.

\section{Les "Témoignages» ("Zeugnisse») et la question des équivalents européens du concept de Märchen}

Dans l'esprit de cette dernière remarque sur l'équivalent espagnol du terme retenu pour désigner le genre, voyons si les témoignages cités servent aussi aux Grimm pour dresser une sorte de vocabulaire européen correspondant à leur concept de Märchen. L'inclusion d'un passage contenant telle ou telle expression étrangère pour désigner ce qui équivaudrait à Märchen signifierait d'une certaine façon que le terme en question en serait la version étrangère autorisée par les Grimm.

Pour l'espagnol, on constate que face à une occurrence de «récits de vieilles» (cuentos de viejas; $\mathrm{n}^{\circ} \mathrm{I} 6$ ) et une de "récits d'enfants» (cuentos de niños; $\mathrm{n}^{\circ} 2 \mathrm{I}$ ), le terme "conseja» que nous venons d'évoquer apparaît dans les trois témoignages de cette sphère linguistique ( ${ }^{\text {os }}$ I4, I6 et 2I). Il s'agit d'un mot qui est lié au substantif consejo dont l'étymologie remonte au latin consilium, qui peut signifier "conseil, avis, assemblée, délibération». Conseja vient du pluriel consilia, ce pluriel accentuant la morale qui généralement conclut ce type de discours qu'on pourrait désigner par l'expression «récit moral» (cuento moral) ${ }^{56}$. Ce terme semble être choisi par les Grimm. On perçoit un lien fort avec les Märchen dans le témoignage $\mathrm{n}^{\circ}$ 2I, bien que cela ne soit pas explicité. Dans la citation de Quevedo, la «maîtresse du curé " (manceba del abad) est mentionnée et il est dit : "et honni soit celui qui l'irait chercher ainsi que la maîtresse du curé» (y el mal para quien le fuere a buscar y para la manceba del $a b a d^{57}$ ). C'est là une variation d'une formule typique des débuts de contes : "Il y avait un jour ce qu'il y avait, que le bien qui vient soit pour tout le monde et le mal pour la maitresse du curé» (Érase que se era, el bien para todos sea y el mal para la manceba del abad ${ }^{58}$ ).

Alors que pour l'espagnol, un terme semble privilégié, pour l'anglais une certaine variété tourne autour de deux épicentres : nursery (nursery-

56. J. Corominas, Breve diccionario etimológico, 2000, p. I67.

57. J. et W. Grimm, KHM, I856, p. 277 / H. Rölleke, KHM, 20IO, III, p. 300.

58. I. Arellano, Francisco de Quevedo, I99I, p. 545 n. 94. 
tale ou nursery-literature) et popular (popular fiction, popular story, popular legends and traditions). Mais dans les témoignages dans d'autres langues, on distingue des concentrations sur un mot en particulier. Ainsi, pour le français, le mot conte accompagné de spécifications comme conte de fées, conte populaire, conte naï; pour l'allemand, l'ancien mare avec ses dérivés, mais aussi le terme contemporain Märchen; et du côté du latin, le terme privilégié dans les citations rapportées par les Grimm est fabula, ou le diminutif fabella. En ce qui concerne le grec ancien, le choix est tombé sur le substantif « $\mu \hat{v} \theta 0 s$ ». L'exemple du grec (témoignages ${ }^{\text {os } ~ I ~ a ̀ ~} 3$ ) mérite d'être examiné d'un peu plus près.

Si pour le $\mathrm{n}^{\circ} 2$ avec Strabon, toute une phrase est citée en grec, pour les autres deux, sur Aristophane ( $\left.\mathrm{n}^{\circ} \mathrm{I}\right)$ et Plutarque $\left(\mathrm{n}^{\circ} 3\right)$, seul le terme " $\mu \hat{v} \theta o s$ » est donné en grec, procédé qui le met en avant. L'importance donnée au terme se perçoit aussi d'une autre façon au ${ }^{\circ}{ }^{\mathrm{I}}$ où est mentionné d'abord le « $\mu \hat{v} \theta 0 \varsigma$ » que raconte le «chœur des vieillards» (Chor der Greise $\left.{ }^{59}\right)$. Vient ensuite l'antistrophe du chœur des femmes, "où l'expression [ $\mu \hat{v} \theta o s]$ se répète" (wo der Ausdruck sich wiederholt ${ }^{60}$ ). Cette indication des Grimm marque l'intérêt qu'ils portent au fait que ce soit $\mu \hat{v} \theta o s$ et non un autre mot qui est utilisé. Il serait trop tôt pour expliquer cette préférence des Grimm pour le substantif $\mu \hat{v} \theta o s$. Ils auraient tout aussi pu choisir des termes

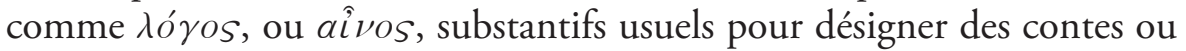
des fables. Le lien avec le terme moderne mythos/mythe et par conséquent avec l'idée de mythologie et de légende (au sens de Sage) semble probable.

\section{Une conclusion provisoire}

De nombreuses questions restent ouvertes. Il faudrait étudier de près tous les passages cités par les Grimm, tenir compte de leurs co-textes et, bien sûr, de leurs contextes. Il faudrait chercher les éditions ou traductions qu'ils utilisent, car les références données dans les «Témoignages» n’apparaissent pas dans le catalogue établi de leur bibliothèque ${ }^{61}$. Y a-t-il des informations dans leur correspondance? Autant de questions comme cette énigme sur laquelle U. Heidmann a attiré mon attention, celle de l'épigraphe de la dernière édition parue du vivant des Grimm (1856/1957) :

Sage vergeht nie ganz, die verbreitete, welche der Völker redende Lippe umschwebt: denn sie ist unsterbliche Göttin. Hesiod 763.

59. J. et W. Grimm, KHM, I856, p. 273 / H. Rölleke, KHM, 20IO, III, p. 297.

6o. Ibid. / Ibid.

6I. F. Krause, Die Bibliothek der Brüder Grimm, 1989. 
La légende ne disparaît jamais entièrement, une fois dispersée, elle plane sur les lèvres des peuples : car elle est une immortelle déesse. Hésiode 763.

D'où vient cette traduction ? Pourquoi n'est-elle pas «fidèle» au texte grec? Car, le passage grec qui lui correspond est le suivant :

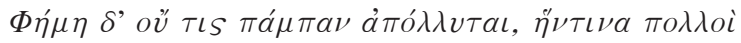

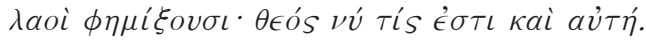

Aucune réputation ne meurt complètement quand de nombreux peuples l'ont divulguée : c'est une sorte de déesse, elle aussi ${ }^{62}$.

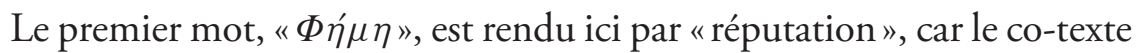
de tout le passage porte sur la bonne et la mauvaise réputation. Ce sens est important quand on considère le contexte du passage cité : Les Travaux et les jours sont un poème didactique, mais ils sont aussi une réaction d'Hésiode à son frère Persès, avec lequel il est en conflit pour des questions d'héritage. Les Grimm, pourtant philologues, ignoraient-ils ces données? Ou utilisent-ils, à leur manière, comme ils le font avec les "Zeugnisse» et les auteurs mentionnés dans la section "Littérature» ou dans les annotations, le matériel qu'ils mettent à disposition? Leur discours scientifique, aux inexactitudes manifestes, poursuit-il un autre but que la scientificité : celui d'une construction et d'une légitimation de leur projet?

En tout cas, ils ont réussi à transformer leur réputation en légende, leur « $\Phi \dot{\mu} \mu \eta$ » est devenue «Sage». Ou est-elle devenue Märchen? Car dans leur dictionnaire, pour le terme "Märe», des rapprochements sont faits avec "Ruf», "Gerücht», "fama», "rumor» ${ }^{63}$. Si, comme le propose Sennewald, les KHM sont aussi un "roman philologique» (philologischer Roman ${ }^{64}$ ), il convient de donner une place à ce genre de questions induites par des éléments que les Grimm avancent eux-mêmes. C'est pourquoi je leur cède plus directement la parole en proposant une traduction du début de la section "Literatur», celle qui porte sur des auteurs aussi importants que Perrault, D’Aulnoy, Basile, Straparola et ceux qu'ils qualifient d' «imitateurs». Cela justifie, en soi, le choix de rester au plus près des remarques, même les plus surprenantes, des Grimm.

62. Hésiode, Les travaux et les jours, v. 762-763; édition de F. Solmsen, I970.

63. J. et W. Grimm, Deutsches Wörterbuch, 1854 .

64. J. E. Sennewald, ouvr. cité, p. 72 et 185. 


\section{Traduction de travail du début de «Literatur»}

La traduction de travail que je propose n'est pas une traduction littéraire achevée; je ne cherche pas à écrire un français parfaitement correct, voire élégant, mais à rendre le plus fidèlement possible le texte allemand. Je me suis efforcée de rester aussi proche que possible de la structure de la phrase allemande, car l'emplacement d'un élément au sein de l'énoncé peut également constituer un effet de sens. Quand j'ai été obligée d'ajouter des éléments pour la lisibilité du français, je les ai signalés par ce signe : $<>$. Quand j'ai dû ajouter des notes, j'ai essayé d'en limiter l'usage. Pour les distinguer de celles des Grimm, j'ai conservé l'astérisque pour les notes originales et numéroté les miennes. Ces notes s'efforceront de clarifier certains choix de traduction, en donnant par exemple au lecteur le mot traduit aussi en allemand. Les notes fournissent des informations qui me semblaient pertinentes, notamment quand les Grimm donnent un titre d'ouvrage qui ne correspond pas exactement au titre effectif.

La question des titres est assez complexe, aussi bien pour les ouvrages que pour les contes : les Grimm donnent tantôt le titre original, que ce soit en allemand ou dans une langue étrangère, tantôt une traduction allemande de textes non germanophones. Pour garder cet effet dans la traduction, j'ai combiné les italiques et les petites majuscules. Sont mis seulement en italiques les titres donnés par les Grimm en allemand. Je les ai traduits en français, mais quand il s'agissait de traductions par les Grimm eux-mêmes de titres en langue étrangère, j'ai rajouté entre crochets [ ] leur version allemande, qui ne correspond précisément pas toujours au titre original. La combinaison des italiques et des petites majuscules a été choisie pour les titres donnés par les Grimm en langues étrangères; je les ai également laissés dans la langue dans laquelle ils étaient chez les Grimm.

Une dernière remarque préalable, de type formel : les chiffres en gras, soulignés et entre parenthèses carrées [ ] renvoient aux pages du fac-similé des Grimm de I856; ceux qui sont donnés entre accolades \{\} renvoient à l'édition de référence de Heinz Rölleke. Il me semblait utile de donner la possibilité de se repérer dans les deux versions.

Tout a été fait pour rendre accessible un texte qui a une valeur stratégique évidente et qui est, à ce titre, d'une importance capitale pour le projet des Grimm. Dans ce sens, cette traduction inédite vient appuyer la démonstration de l'étude d'Ute Heidmann. 


\section{$\{309\} /[285]$ Les Nuits de Straparola ${ }^{65}$}

En l'an I550, parut à Venise la première partie d'un recueil, par la suite souvent réimprimé (deuxième édition en 1554 ), de récits, facéties ${ }^{66}$ et énigmes, réunis d'une façon semblable à celle de Boccace et présentés sous le titre Treize nuits plaisantes [Dreizehn ergötzliche Nächte] (TREDICI PIACEVOLI NOTTI). Il contient au total 74 pièces, réparties en 13 nuits, parmi lesquelles il y a aussi 2I contes ${ }^{67}$. L'auteur Giovan Francesco Straparola, de Caravaggio dans la région milanaise, doit avoir vécu entre la fin $\mathrm{du} \mathrm{Xv}$ et le milieu du $\mathrm{XVI}^{\mathrm{e}}$ siècle, puisqu'une édition de ses poèmes est parue déjà en I508 à Venise; on ne peut pas en dire plus, car ni sa date de naissance ni celle de sa mort ne sont connues, et rien sur sa vie n’a été noté. Il a puisé la matière de ses Nuits [Nächte] à divers endroits, point sur lequel on trouve des preuves ${ }^{68}$ chez Dunlop (Liebrecht 283, 284, 494-497); or cela ne vaut pas pour les contes, qui ont été recueillis à partir de la tradition orale. Cependant, un des contes $(\mathrm{I} 2,3)$ est tiré de Morlini et a été conservé sans modification; un autre $(5,7)$ montre une parenté avec le premier. Dans les récits de ce dernier, souvent indécents, rédigés en latin et qui viennent d'être réédités (Novellae, fabulae, comoedia, Paris, I855), on ne trouve par ailleurs rien de merveilleux ${ }^{69}$; cf. Liebrecht à Dunlop <pages> 494-498. Le cadre et la présentation chez Straparola ne sont toutefois pas équivalents et, même dans les meilleures pièces, ils ne sont pas d'une exactitude extraordinaire : cependant, certaines choses sont racontées de façon agréable, naturelle et non sans finesse, d'autres au contraire <le sont de façon> non seulement indécente, mais vont jusqu'à l'obscène impudique et sont sans vergogne, au point de ne pas pouvoir être excusées par les mœurs naturelles et libres de l'Italie et de cette époque-là. [286] C'est pourquoi le livre fut inclus en 1605 dans l'index des écrits interdits et qu'on en prépara une édition abrégée et purgée. Assez dénués de cette saleté, les contes constituent de toute façon la meilleure partie de tout l'ouvrage. Comme il est dit dans le prologue de la deuxième partie (avant la sixième Nuit), Straparola les a «reçus de la bouche de dix jeunes femmes»; et il y est clairement expliqué qu'ils ne sont pas de sa propriété. Les meilleures $\{3 \mathbf{3} 0\}$ preuves littéraires sont offertes par une traduction allemande (Die Nächte des Straparola von Caravaggio, Vienne I79I. 8, deux parties), dans le prologue de laquelle on

65. La traduction rapporte les noms propres tels qu'indiqués par les Grimm.

66. Le terme «facétie» est ici utilisé pour traduire le mot allemand "Schwank».

67. Le mot «conte» traduit ici l'allemand "Märchen».

68. Le substantif "preuves» traduit l'allemand "Nachweisungen».

69. L'adjectif «merveilleux» est ici utilisé pour traduire l’allemand "märchenhaft». 
a en effet reproduit un essai sur Straparola provenant d'un legs manuscrit du savant Mazzuchelli qui porte sur la continuation de sa grande ouvre; en même temps, on trouve au même endroit ce que disent sur lui Quadrio, dans son histoire de la poésie, et d'autres <auteurs>. Pour les éditions et les traductions, il faut consulter Bartol. GAMBA DELLE NOVELLE ITALIANE IN PROSA BIOGRAFIA (Florence I835) p. I6o s. et Ebert bibliogr. Lexikon 2, 847. Probablement, il existait au Xvi ${ }^{\mathrm{e}}$ siècle une traduction allemande de Straparola, puisque Fischart, dans le Gargantua p. 7, mentionne les «histoires de Straparola». Bretschneider pense à une <traduction> de I679. 8 dans l'annonce d'une nouvelle édition du Gargantua. Il n'est pas nécessaire de donner un extrait des contes, puisqu'ils ont été rendus accessibles par Friedr. Wilh. Val. Schmidt (Märchen-Saal. Premier tome. Berlin I8I7) dans sa bonne traduction, accompagnée d'annotations diligentes et estimables et qui surpasse de loin la traduction viennoise qui ne contenait que les six premières Nuits [Nächte]. Dommage qu'il utilise sans le savoir une édition défectueuse $^{70}$ (Venise i608). Nous nous contentons donc de relever les contes dans l'ordre de l'original que Schmidt ne respecte pas et d'indiquer sous forme d'extraits ${ }^{71}$ seulement ceux qu'il ne mentionne pas. Nous avons devant nous l'édition complète (Venise 1573) et une traduction française (Lyon I6II, inconnue de Mazzuchelli) lui correspondant et même encore plus complète, car il n'y manque pas le bref prologue du deuxième volume.

I, I. Les trois interdits du père [Die drei Verbote des Vaters]. Schmidt, p. 70.

2. L'imposteur [Der Gauner]. Il résout trois tâches. D'abord, il vole à Probst le lit sur lequel il est couché. Ensuite, il emmène le cheval sur lequel est assis le valet d'écurie sans qu'il s'en rende compte. [287] Enfin, il met un autre homme spirituel dans un sac. Dans la trad. viennoise p. 32, mais incomplètement. Chez Schmidt, délaissé à tort, car cela se trouve aussi dans l'édition défectueuse, même si c'est abrégé. Voir le conte allemand du Maître-voleur $\left[\right.$ Meisterdieb] $\mathrm{n}^{0} 192^{72}$.

3. Maître Scarpacifico [Meister Scarpacifico] (Schmidt, p. 133) est trompé et $\{\mathbf{3} \mathbf{I I}\}$ trompe à son tour. Apparenté au conte allemand du Petit paysan [Bürle] n ${ }^{\circ}$ 6r.

70. Le terme "défectueux» traduit l'allemand «castriert».

7I. Le substantif «extrait» traduit le mot allemand «Auszug».

72. Sauf dans le cas où les Grimm donneraient ici une version abrégée ou légèrement remaniée des titres de leur recueil, les titres sont rendus en français selon la traduction de N. Rimasson-Fertin, Contes pour les enfants et la maison, 2009; le titre allemand tel que le donnent les Grimm ici est ajouté entre crochets. 
4. La fille dans le cercueil [Das Mädchen im Schrein] (Schmidt, p. II5). Un joli conte en lui-même auquel correspondent seulement dans certains détails des contes italiens et allemands. Mais comp. Gesamtabenteuer 3, CLVI de Hagen.

II, I. Roi porc [König Schwein] (Schmidt, p. 249). En allemand Hansmon-hérisson [Hans mein Igel] $\mathrm{n}^{\circ}$ Io8.

III, I. Pierre le sot ${ }^{73}$ [Der dumme Peter] (Schmidt, p. 23I). Est plus merveilleux <le> PERVONTO dans le PENTAMERONE $\mathrm{n}^{\circ} \mathrm{I}, 3$.

2. Le cheval magique [Das Zauberpferd] (Schmidt, p. I). En allemand, y est apparenté Le serpent blanc [Die weisse Schlange] $\mathrm{n}^{\circ} \mathrm{I} 7$.

3. Le serpent [Die Schlange] (Schmidt, p. 24).

4. Le cadeau des trois animaux [Das Geschenk der drei Thiere] (Schmidt, p. 158$)$.

5. Le véritable [Der Wahrhafte] (Schmidt, p. I47).

IV, I. Princesse en cavalier [Prinzessin als Ritter] (Schmidt, p. 195).

3. Les trois enfants du roi [Die drei Königskinder] (Schmidt, p. 44). Allemand Les trois petits oiseaux [De drei Vügelkens] $\mathrm{n}^{\circ} 96$.

V, I. L'homme des bois [Der Waldmann] (Schmidt, p. 92). Allemand Jean-de-fer [Der Eisenhans] n ${ }^{\circ} 136$.

2. La poupée (poavola). Manque chez Schmidt. Dans la trad. viennoise 2, 97-IO5, où cependant on fait de la poupée une pie qui s'assoit sur l'épaule du roi et qui s'y accroche si fortement que seulement la plus jeune fille du roi réussit à l'en déloger. Dans le PENTAMERONE $\mathrm{n}^{\circ} 4 \mathrm{I}$, au lieu d'une poupée, il s'agit d'une oie, pour le reste, correspondant en tout.

VII, 5. Les trois frères [Die drei Brüder] (Schmidt, p. 262). Chez Morlini $\mathrm{n}^{\circ} 79$. Plus complet et meilleur dans le PENTAMERONE Les cinq fils $(5,7)$. En allemand Les quatre frères [Die vier Brüder] $\mathrm{n}^{\circ} \mathrm{I} 29$.

VIII, 5. L'apprenti sorcier [Der Zauberlehrling]. En allemand Le Voleur [Der Gaudeif] n ${ }^{\circ}$ 68. [288] Manque dans l'édition défectueuse de Straparola, donc aussi chez Schmidt (où suit immédiatement la facétie avec les deux médecins), dans l'édition complète, la $6^{\mathrm{e}}$ fable.

Lactantius, magicien à l'insu de tous, pratique ouvertement le travail de couturier; son apprenti l'épie et n'a plus envie de coudre, raison pour laquelle son père le ramène chez lui. Le magicien, toutefois, le reprend, \{3I2\},

73. En fait, chez Straparola, il est question de "Pietro Pazzo", donc de "Pierre le fou» ce qui en allemand donnerait «Der wahnsinnige Peter». Or les Grimm parlent ici de "Der dumme Peter», d'où la traduction française «Pierre le sot». 
mais il doit alors accomplir de viles besognes, de sorte que le père vient de nouveau le chercher. Comme ils sont pauvres, le jeune homme dit : «Père, je veux me transformer en un joli cheval, vendez-moi, mais gardez les brides et ne me donnez pas avec, sinon je ne pourrai plus revenir.» Lactantius reconnaît le cheval, l'achète au père et le convainc de lui donner aussi les brides. Alors, il l'attache, le bat et le maltraite. Mais les filles du magicien le conduisent une fois près de l'eau; là il se transforme immédiatement en un petit poisson et plonge dans l'eau. Le magicien court après lui, il se transforme en un poisson carnassier qui poursuit le petit. Celui-ci, toutefois, sous forme d'un rubis enchâssé dans un anneau d'or, saute dans la corbeille de la fille du roi, qui est en train de cueillir des cailloux. Elle l'emmène, il se présente à elle sous son véritable aspect de beau jeune homme qu'elle apprend à aimer et qu'elle garde auprès d'elle sous forme d'un anneau. Le vieux roi tombe malade, Lactantius, métamorphosé en médecin, le soigne, et demande comme récompense seulement un anneau de rubis que devrait avoir sa fille, car il sait bien de quoi il s'agit. Elle ne veut pas le donner; quand à la fin elle y est obligée, le jeune homme lui dit de jeter, devant le magicien, l'anneau contre le mur. Aussitôt que l'anneau tombe à terre, il se transforme en une grenade qui éclate et ses grains roulent en tous sens. Le maître se transforme en un coq pour picorer les grains; mais un $<$ des grains $>$ dissimulé reste invisible. Ce petit grain se transforme en un renard qui attrape et mort au cou le coq, qu'il tue. Le roi lui accorde par la suite sa fille pour épouse.

$\mathrm{X}$, 3. Les animaux fidèles [Die treuen Thiere] (Schmidt, p. 215). Plus complet en allemand, Les deux frères [Die beiden Brüder] $\mathrm{n}^{\circ} 60$. Dans le PENTAMERONE, le $\mathrm{n}^{\circ} 7$ est assez ressemblant.

XI, I. Le chat [Der Kater] (Schmidt, p. I80). Dans les «Fragments» en haut $\mathrm{n}^{\circ} 4$ [289] Gagliuso dans le PENTAMERONE 2, 4. Le chat botté [Der gestiefelte Kater] chez Perrault.

2. Le sot ${ }^{74}$ [Der Dummling]. Manque dans l'édition défectueuse et chez Schmidt.

Bertuccio, un sot, doit recevoir sa fortune paternelle seulement à ses trente ans, mais sa mère doit lui verser trois cents ducats s'il les demande. Il s'en fait donner cent, part avec et trouve un homme qui $\{3 \mathbf{I 3}\}$ a tué un autre homme, et il le bat encore alors qu'il est déjà mort. Par compassion, le sot lui donne quatre-vingt pièces d'or et rachète ainsi le cadavre; il utilise le reste pour le faire enterrer honnêtement. Sa mère se fâche de sa

74. Le terme «sot» traduit l'allemand «Dummling»; «sottise» traduit «Dummheit». 
sottise, mais lui exige les autres deux cents ducats, part et libère, avec cet argent, la fille du roi des mains de brigands. Une fois qu'il l'a ramenée à la cour de son père, elle lui dit qu'elle ne veut épouser personne d'autre que lui : quand il arrivera, il devra avoir la main droite sur la tête; alors, elle le reconnaîtra ${ }^{75}$. Il part sur un cheval misérable; sur la route, il rencontre un chevalier qui lui donne son beau cheval et ses habits magnifiques, contre lesquels le sot doit lui promettre qu'à son retour, il partagera avec lui tout ce qu'il aura acquis. Le beau chevalier plaît au roi et Bertuccio obtient sa bien-aimée. Sur le chemin du retour, il rencontre le chevalier qui exige à présent la moitié du tout. Le sot partage aussitôt tout ce qu'il a reçu pour le mariage. Alors le chevalier exige aussi la moitié de la femme. "Comment cela devrait-il se faire?» demande Bertuccio. "Nous devons la découper.» "Dans ce cas, prends-la en entier, dit le sot, je l'aime trop pour pouvoir accepter.» Alors le chevalier étranger dit "garde tout et reprends tout, je suis l'esprit du mort assassiné et j'ai voulu te récompenser pour ce que tu as fait pour moi".

XII, 3. Bon conseil [Guter Rath] (Schmidt, p. I88). Un coq conseille de battre une femme indocile pour la guérir de son opiniâtreté. Le conte est emprunté à Morlini n ${ }^{\circ} 7 \mathrm{I}$. Avec une autre introduction, il est aussi raconté dans les Mille et Une Nuits [Ioor Nacht] (I, 36 s.). Une version ${ }^{76}$ spécifiquement serbe, chez Wuk n ${ }^{\circ} 3$, est meilleure encore et très simple, toutefois, une < version $>$ africaine chez Kölle p. I43; voir ci-dessous. [290]

XIII, 6. Les bons jours [Die guten Tage] (Schmidt, p. 246). Apparenté au conte allemand du Docteur-je-sais-tout [Doktor Allwissend] $n^{\circ} 98$.

\section{$\{314\} /[290]$ Le Pentamerone de Basile}

Au XVII ${ }^{\mathrm{e}}$ siècle suivant parut à Naples, en dialecte napolitain, un recueil plein de contes, appelé, en imitation du DECAMERONE, le PENTAMERONE, par Giambattista Basile; livre presque complètement inconnu à l'étranger,

75. Le futur dans la traduction rend le discours indirect avec Konjunktiv (subjonctif) dans le texte allemand. La logique du début de la phrase allemande pose d'ailleurs problème : après avoir évoqué la libération de la princesse, les Grimm disent littéralement "qu'on vient chercher " la princesse (abgeholt wird) à la cour du roi, où précisément elle tient ces propos rapportés au style indirect. Or la scène laisse clairement entendre que la princesse est ramenée à la cour, où Bertuccio la laisse pour revenir la chercher ensuite. Pour conserver la cohérence de l'histoire, j’ai donc préféré dire qu'elle «est ramenée» à la cour de son père.

76. Le terme "version" traduit l'allemand "Auffassung".

* Römische Studien 3, <pages> 316. 317. 462. 475. 476. 536. 539. Les différentes rares éditions que Fernow a recueillies se trouvent maintenant à la bibliothèque grand-ducale de Weimar. 
le premier à y penser parmi nous a été Fernow*. L'auteur (appelé par anagramme aussi Gian Alesio Abbatutis), vécut au début du XvII ${ }^{\mathrm{e}}$ siècle. Après avoir passé le début de sa jeunesse sur l'île de Crète, il fit la connaissance des Vénitiens et fut accueilli dans la academia degli stravaganti. Il suivit sa sœur Adriana, une célèbre chanteuse, à Mantoue et entra aux services du duc auprès duquel il jouit de quelque faveur. Il voyagea beaucoup en Italie, revint aussi à Naples où il dut mourir autour de l'an I637*. La première édition du PENTAMERONE que l'on connaisse a probablement été précédée, puisqu'elle est de l'an 1637 , d'une édition antérieure ${ }^{77}$, complètement épuisée. La série de tirages que le livre a connus depuis ${ }^{* *}$ lui assurerait déjà d'avance une certaine valeur; ce recueil de contes [29I] à lui seul a longtemps été le meilleur et le plus riche de tous ceux qu'un peuple ait produits. Non seulement <parce> qu'à l'époque la tradition était mieux préservée, \{3I5\} mais l'auteur possédait aussi, à côté de la connaissance exacte du dialecte, une habilité particulière pour se l'approprier. Le contenu est presque sans lacune, et le ton, au moins des Napolitains, est parfaitement trouvé; en cela il a également l'avantage sur Straparola qui visait une façon de raconter habituelle <et> cultivée et ne savait pas changer de ton. En ce sens, on peut considérer ce recueil de 50 contes (l'introduction et la fin y compris), avec leur riche contenu, comme fondamental ${ }^{78}$; car, même si <ce recueil > était inconnu à l'étranger et n'a même pas été traduit en français, il a toutefois, relativement à la tradition, cette réputation. Deux tiers < des contes $>$ se retrouvent dans leurs traits principaux en allemand et sont de nos jours encore vivants. Basile ne s'est permis aucun changement, à peine un ajout important, et cela donne aussi à son œuvre une valeur particulière. Il n’a pas utilisé son devancier Straparola; il ne l'a probablement même pas connu. Les deux n'ont que quatre pièces en commun $\left(\mathrm{n}^{\mathrm{os}} 3\right.$, I4, 4I, 45 chez Straparola

* Eustache d'Afflitto, Memorie degli scrittori del regno di Napoli, Naples, I794, I, <pages> 68-72. Selon Liebrecht 2, <page> 322, son titre complet était Giovan Battista Basile, Cavalier, Comte de Torrana et Pfalzgraf. Son portrait se trouve, comme l'indique Mazzuchelli, dans l'œuvre Le glorie degli incogniti, p. 209.

77. Transformation passive de la phrase active allemande en français.

** Selon Fernow et Galiani (Del dialetto napoletano, Naples, 1779), il est paru ensuite à Naples en I645, I674, I7I4, I722, I788 (Collezione di tutti li poeti in lingua napoletana, t. 20 et 2I), à quoi s'ajoute une <édition> de 1749 nulle part mentionnée que possédait Cl. Brentano. Voir Bartolommeo Gamba, Delle Novelle Italiane, p. I7I-I72 et Brunet, MANUEL DU LIBRAIRE (Paris, I842), I, <page> 260. À Rome en 1679 selon Fernow, puis à Naples en 1754 avec des gravures selon Brunet et Ebert. Le tout in I2. En outre, parut une traduction abrégée, en italien courant, jugée par Liebrecht très mauvaise, Naples 1769. I794 et une autre <édition> en dialecte bolognais Bolog. I742.

78. L'allemand «Grundlage» signifie «fondement» et sera rendu ainsi dans la suite de la traduction; ici, cependant, il est adapté au français et traduit par «fondamental». 
3, I; IO, I ; 5, 2; 7, 5) et il ressort clairement de la comparaison qu'il écrivait de façon autonome. Dans cette perspective, le conte de la poupée est <un cas> étrange (5, I chez Straparola 5, 2) : Basile le raconte, pour le reste $<$ de façon> assez concordante, avec une oie, ce qui en effet convient moins, et visiblement Straparola a <la version la> plus correcte, comme d'ailleurs d'autres traits supplémentaires; la différence s'explique toutefois par la ressemblance entre les deux mots, confondus dans la tradition orale : papara oie et pipata poupée*. Basile a raconté complètement selon l'esprit [292] d'un peuple vif, spirituel et badin, \{316\} faisant continuellement allusion aux mœurs et aux coutumes, et même à l'histoire ancienne et à la mythologie dont la connaissance est de toute façon répandue chez les Italiens. Là réside l'opposition avec le style calme et simple des contes allemands. Il est extrêmement fécond en expressions figurées et proverbiales et en tournures spirituelles qu'il a toujours sous la main et qui touchent juste; assez souvent, l'expression aussi est, à la mode du pays, audacieuse, libre et directe et en ce sens elle choque notre sentiment, p. ex. il serait difficile de raconter chez nous dans son intégralité ce conte sur la poupée précisément, mais on ne peut pas le qualifier d'indécent comme celui de Straparola. Une certaine exubérance et effusion du discours lui sont naturelles, p. ex. dans le $23^{\mathrm{e}}$ conte <où> la lamentation de Renza se prolonge pendant deux pages, il s'agit toutefois seulement de l'attrait, propre aux peuples du Sud, pour l'expression toujours nouvelle du <même> sujet, mais non pas d'un sujet pauvre qu'il s'agirait de couvrir. Selon l'opinion de Liebrecht $(<$ dans sa traduction de> Dunlop <pages> 517-518) il a en cela imité Rabelais. Puisque la profusion d'allégories 79 est généralement mise en avant par le badinage et l'esprit, on peut utiliser ici les <contes les> plus étranges et ridicules, sans qu'ils soient plats; ainsi, p. ex. dans le $23^{\mathrm{e}}$ conte, l'amant crie à $<$ sa $>$ bien-aimée : «Adieu, ô registre de tous les privilèges de la nature, archive de toutes les grâces accordées par le ciel, table décrite avec tous les titres

* Un contre argument à cela a été apporté par Liebrecht 2, < page > 260 et < dans sa traduction de> Dunlop <page> 517. Je [Wilhelm Grimm] ne prétends pas que Basile ait intentionnellement transformé pipata en papara, je dirais même plus que c'est la tradition vivante qui l'a fait. Une poupée de chiffons pouvait être utilisée comme meilleur instrument de nettoyage qu'une oie, dont la survie, après que <son> cou a été retourné, est pour le moins peu probable. De plus, Rabelais demande un OISON DUMETÉ, comme on le raconte à propos du sourd qui aurait utilisé à cet usage une petite oie possédant encore ses plumules. La poupée était un être du genre lutin, apparenté au fameux petit homme des ducats, et la version me semble être la plus originale [traduction pour : "ursprünglichere»].

79. Le terme «allégories » traduit l'allemand « Gleichnisse». 
de la beautée ${ }^{80}$. "Un certain $<$ degré de $>$ développement ${ }^{8 \mathrm{r}}$ du héros apparaît dans le $38^{\mathrm{e}}<$ conte> ; le $32^{\mathrm{e}}$ n'est pas vraiment merveilleux, mais ressemble plutôt à un poème didactique; le $20^{\mathrm{e}}$ est une facétie, et le $26^{\mathrm{e}}$ est le plus faible en ce qui concerne le contenu et l'exécution.

La ressemblance que le conte $L O D R A G O N E(4,5)$ entretient avec la légende ${ }^{82}$ de Siegried mérite une remarque particulière. La naissance secrète du garçon, comme le service humble auprès du cuisinier, rappellent l'enfance de Siegfried. Ensuite, nous le voyons secouru par un oiseau serviable qui rappelle les oiseaux dont le Sigurd nordique comprend le langage et desquels il reçoit un conseil, qu'il accepte. La reine courroucée rencontre ensuite Brünhild et est en même temps Reigen qui incite au combat avec le dragon; le dragon est ici aussi le frère de la reine et sa vie est liée à la sienne. Elle veut elle aussi être badigeonnée de [293] son sang, de même que Reigen veut le sang du cœur de Fafner.

Puisqu' une appréciable traduction de Felix Liebrecht est parue, pourvue d'annotations savantes et d'un essai (Breslau I846 en deux volumes), <et qu'est parue $>$ aussi, peu après, une < traduction $>$ en anglais de John Edward Taylor (Londres I848), ainsi il n'est pas nécessaire de donner un extrait, de sorte qu'on se contente ci-après de survoler les contes du PENTAMERONE et du recueil allemand qui coïncident dans leur totalité ${ }^{83}$.

\begin{tabular}{|c|c|c|c|c|}
\hline$(\mathrm{I}, \mathrm{I})$ & I & $\begin{array}{l}\text { L'homme sauvage } \\
{[\text { Der wilde Mann }]^{84}}\end{array}$ & $\begin{array}{l}\mathrm{n}^{\circ} \\
36\end{array}$ & $\begin{array}{l}\text { Petite-table-sois-mise } \\
\text { [Tischchen deck dich] }\end{array}$ \\
\hline$(\mathrm{I}, 2)$ & 2 & $\begin{array}{l}\text { La branche de myrte } \\
\text { [Der Heidelbeerstrauch] }\end{array}$ & 76 & L'oillet [Die Nelke] \\
\hline$(I, 4)$ & 4 & Vardiello [Vardiello] & 59 & $\begin{array}{l}\text { Frédéric et Lisette [Frieder } \\
\text { und Catherlieschen] }\end{array}$ \\
\hline
\end{tabular}

8o. L'expression napolitaine "adio», qui dans le contexte du passage de Basile signifie «bonjour» (édition de M. Rak, Basile, I999, p. 502), est traduite de manière erronée par «Lebewohl» (adieu) dans la version allemande utilisée par les Grimm; j'ai gardé leur traduction, d'où le français « adieu ".

8I. Le substantif "développement" traduit l'allemand "Ausbildung».

82. Le terme «légende» traduit l'allemand "Sage».

83. Les titres que les Grimm attribuent aux récits de Basile ne correspondent pas toujours à ceux en napolitain. La présente traduction rend les intitulés tels que les Grimm les fournissent. En cas de changement de titre par les Grimm, une note ajoute la version napolitaine (selon l'édition bilingue napolitain-italien par M. Rak, Basile, 1999), avec la traduction par F. Decroisette, Giambattista Basile, 2002.

84. Pour : "Der wilde Mann»; mais Basile : "Lo cunto dell'uerco", Decroisette : "Le conte de l'ogre». 


\begin{tabular}{|c|c|c|c|c|}
\hline$(I, 5)$ & 5 & La puce [Der Floh] & 7I & $\begin{array}{l}\text { Six à travers le monde } \\
\text { [Sechse durch die Welt]. } \\
\text { Fragment } 2 \text { du Pou [Laus] }\end{array}$ \\
\hline$(\mathrm{I}, 6)$ & 6 & $\begin{array}{l}\text { La petite chatte des cendres } \\
\text { [Aschenkätzchen] }\end{array}$ & $2 \mathrm{I}$ & $\begin{array}{l}\text { Cendrillon } \\
{[\text { Aschenputtel }]}\end{array}$ \\
\hline$(\mathrm{I}, 7)$ & 7 & $\begin{array}{l}\text { Le marchand } \\
{[\text { Der Kaufmann }]}\end{array}$ & 60 & $\begin{array}{l}\text { Les deux frères } \\
\text { [Die zwei Brüder] }\end{array}$ \\
\hline$(\mathrm{I}, 8)$ & 8 & $\begin{array}{l}\text { Face de chèvre } \\
\text { [Das Zeigengesicht }]\end{array}$ & 3 & $\begin{array}{l}\text { L'enfant de Marie } \\
{[\text { Marienkind] }}\end{array}$ \\
\hline$(\mathrm{I}, 9)$ & 9 & $\begin{array}{l}\text { La biche } \\
{[\text { Die Hirschkuh }]^{85}}\end{array}$ & 60 & $\begin{array}{l}\text { Les deux frères } \\
\text { [Die zwei Brüder] }\end{array}$ \\
\hline$(2, I)$ & II & $\begin{array}{l}\text { Petrosinella } \\
\text { [Petrosinella }]\end{array}$ & I2 & $\begin{array}{l}\text { Raiponce } \\
{[\text { Rapunzel] }}\end{array}$ \\
\hline$(2,5)$ & I5 & $\begin{array}{l}\text { Le serpent } \\
{[\text { Die Schlange }]}\end{array}$ & I08 & $\begin{array}{l}\text { Hans-mon-hérisson } \\
\text { [Hans mein Igel] }\end{array}$ \\
\hline$(2,6)$ & I6 & $\begin{array}{l}\text { L'ourse } \\
\text { [Die Bärin] }\end{array}$ & 65 & $\begin{array}{l}\text { Toutes-fourrures } \\
\text { [Allerlei-Rauh }]\end{array}$ \\
\hline$(2,7)$ & I7 & $\begin{array}{l}\text { Le raisin } \\
{[\text { Die Traube }]^{86}}\end{array}$ & 56 & $\begin{array}{l}\text { Le bien-aimé Roland } \\
\text { [Der liebste Roland] }\end{array}$ \\
\hline$(2,8)$ & I8 & La servante de cuisine $e^{87}$ & 53 & $\begin{array}{l}\text { Sneewitchen } \\
{[\text { Blanche-neige }]}\end{array}$ \\
\hline$(2,9)$ & 19 & $\begin{array}{l}\text { Le coffret magique } \\
{[\text { Das Zauberkästchen }]^{88}}\end{array}$ & 88 & $\begin{array}{l}\text { L'alouette } \\
\text { [Löweneckerchen] }\end{array}$ \\
\hline$(2, \mathrm{IO})$ & 20 & $\begin{array}{l}\text { Le compère } \\
\text { [Der Gevatter] }\end{array}$ & 6I & $\begin{array}{l}\text { Le petit paysan } \\
\text { [Das Bürle] }\end{array}$ \\
\hline$(3,2)$ & 22 & $\begin{array}{l}\text { Fille sans mains } \\
{\left[\text { Mädchen ohne Hände }{ }^{89}\right.}\end{array}$ & $3 \mathrm{I}$ & $\begin{array}{l}\text { Jeune fille sans mains } \\
\text { [Mädchen ohne Hände] }\end{array}$ \\
\hline$(3,6)$ & 26 & $\begin{array}{l}\text { Bonne } \\
{[\text { Dienstmagd }]^{9 \circ}}\end{array}$ & 67 & $\begin{array}{l}\text { Les douze chasseurs } \\
\text { [Die zwölf Jäger] }\end{array}$ \\
\hline$(3,7)$ & 27 & $\begin{array}{l}\text { Corvetto } \\
{[\text { Corvetto }]}\end{array}$ & I26 & $\begin{array}{l}\text { Fernand-le-fidèle } \\
{[\text { Ferenand getrü] }}\end{array}$ \\
\hline$(3,8)$ & 28 & $\begin{array}{l}\text { Le sot } \\
{[\text { Der Dummling }]^{91}}\end{array}$ & 7I & $\begin{array}{l}\text { Six à travers le monde } \\
{[\text { Sechse durch die Welt }]}\end{array}$ \\
\hline
\end{tabular}

85. Pour : "Die Hirschkuh»; mais Basile : La cerva fatata, Decroisette : «La biche ensorcelée». 86. Pour "Die Traube», mais peut-être s'agit-il d'une erreur pour "Die Taube», la colombe, ce qui correspondrait mieux au titre de Basile, «La palomma».

87. Pour : "Die Küchenmagd»; mais Basile : «La schivottella», Decroisette : «La petite esclave».

88. Pour : «Das Zauberkästchen»; mais Basile : «Lo catenaccio», Decroisette : «Le verrou».

89. Pour : "Mädchen ohne Hände»; mais Basile : "La Penta Mano-mozza", Decroisette : «Penta-la-manchotte».

90. Pour : «Dienstmagd»; mais Basile : «La Serva d'aglie», Decroisette : «Le bois des aulx».

91. Pour : «Der Dummling»; mais Basile : "Lignorante», Decroisette : «L'ignorant». 


\begin{tabular}{|c|c|c|c|c|}
\hline$(3,9)$ & 29 & $\begin{array}{l}\text { Rosella } \\
{[\text { Rosella }]\{3 \mathbf{I} 8\}}\end{array}$ & 56 & $\begin{array}{l}\text { Le bien-aimé Roland } \\
\text { [Der liebste Roland }]\end{array}$ \\
\hline$(3, \mathrm{IO})$ & 30 & $\begin{array}{l}\text { Les trois fées } \\
{[\text { Die drei Feen }]}\end{array}$ & I3 & $\begin{array}{l}\text { Les trois petits hommes } \\
\text { [Die drei Männlein] }\end{array}$ \\
\hline$(4, I)$ & $3 \mathrm{I}$ & $\begin{array}{l}\text { La pierre du coq } \\
\text { [Der Hahnenstein] }\end{array}$ & IO4 & $\begin{array}{l}\text { Les animaux fidèles } \\
\text { [Die treuen Thiere] }\end{array}$ \\
\hline$(4,3)$ & 33 & $\begin{array}{l}\text { Les trois frères animaux } \\
{[\text { Die drei Thierbrüder }]^{92}}\end{array}$ & I97 & $\begin{array}{l}\text { La boule de cristal } \\
\text { [Die Krystallkugel] }\end{array}$ \\
\hline$(4,4)$ & 34 & $\begin{array}{l}\text { Les sept petites couennes } \\
{[\text { Die sieben }} \\
\text { Speckschwarten] }\end{array}$ & I4 & $\begin{array}{l}\text { Les trois fileuses } \\
{[\text { Die drei Spinnerinnen }]}\end{array}$ \\
\hline$(4,7)$ & 37 & $\begin{array}{l}\text { Les deux gâteaux } \\
{[\text { Die zwei Kuchen }]^{93}}\end{array}$ & 24 & $\begin{array}{l}\text { Dame Holle [Frau Holle] et } \\
\text { I35 La fiancée blanche et la } \\
\text { fiancée noire [Die weisse u. } \\
\text { schwarze Braut] }\end{array}$ \\
\hline$(4,8)$ & 38 & $\begin{array}{l}\text { Les sept colombes } \\
{[\text { Die sieben Tauben] }}\end{array}$ & 25 & $\begin{array}{l}\text { Les sept corbeaux } \\
{[\text { Die sieben Raben] }}\end{array}$ \\
\hline$(4,9)$ & 39 & Le corbeau [Der Rabe] & 6 & $\begin{array}{l}\text { Le fidèle Jean } \\
\text { [Der treue Johannes] }\end{array}$ \\
\hline$(4$, IO $)$ & 40 & $\begin{array}{l}\text { L'orgueil châtié } \\
\text { [Der bestrafte Hochmuth] }\end{array}$ & 52 & $\begin{array}{l}\text { Le roi Bec-de-grive } \\
\text { [König Drosselbart] }\end{array}$ \\
\hline$(5,3)$ & 43 & $\begin{array}{l}\text { Pintosmauto } \\
{[\text { Pintosmauto }]}\end{array}$ & 88 & $\begin{array}{l}\text { L'alouette } \\
\text { [Löweneckerchen] }\end{array}$ \\
\hline$(5,4)$ & 44 & $\begin{array}{l}\text { La racine d'or } \\
{[\text { Die goldene Wurzel }]^{94}}\end{array}$ & & \\
\hline$(5,5)$ & 45 & $\begin{array}{l}\text { Soleil, Lune et Tahia } \\
{\left[\text { Sonne, Mond und Tahia }{ }^{95}\right.}\end{array}$ & 50 & $\begin{array}{l}\text { Rose d'épine } \\
\text { [Dornrörschen }]\end{array}$ \\
\hline$(5,7)$ & 47 & $\begin{array}{l}\text { Les cinq fils [Die fünf } \\
\text { Söhne] }\end{array}$ & I29 & $\begin{array}{l}\text { Quatre frères } \\
\text { [Vier Brüder] }\end{array}$ \\
\hline$(5,8)$ & 48 & $\begin{array}{l}\text { Nennillo et Nennella } \\
{[\text { Nennillo und Nennella }]}\end{array}$ & I5 & $\begin{array}{l}\text { Hänsel et Gretel } \\
{[\text { Hänsel und Gretel] }}\end{array}$ \\
\hline
\end{tabular}

Encore faut-il mentionner que Rosella $(3,9)$ correspond en partie au conte des Trois ceintures [drei Gürtel] dans la Braunschweig. Sammlung (voir ci-dessous), et que les Trois frères animaux [Die drei Thierbrüder] $(4,3)$ correspondent également à un conte chez Musée.

92. Pour : «Die drei Thierbrüder»; mais Basile : "Li tre ri animale», Decroisette : «Les trois animaux rois".

93. Pour : «Die zwei Kuchen»; mais Basile : «Les doie pizzelle», Decroisette : «Les deux petites pizzas».

94. Pour "Die goldne Wurzel»; mais Basile : «Lo turzo d'oro» (turzo : «tronc»), Decroisette : «L'arbre d'or».

95. Basile : «Sole, Luna e Talia», et non pas Tahia, comme l'indiquent les Grimm.

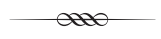




\section{\{318\}/[294] Gesta Romanorum}

C'est le titre d'un recueil rédigé en latin de récits anciens tirés de diverses sources et qui se réfèrent généralement aux actions d'empereurs romains. Il a probablement été écrit au milieu du XIV siècle, on ne peut pas dire avec certitude par qui. L'auteur peut avoir été anglais ou français, <mais> plus probablement, \{319\} puisque y apparaissent des noms allemands de chiens, était-il allemand. On trouve un essai sur lui dans la traduction allemande de Gräße (Dresde et Leipzig I842, deux tomes), où toutes les éditions et traductions sont soigneusement indiquées. Nous ne prenons en considération que les récits qui sont merveilleux et qui en même temps pourraient provenir originairement de la tradition orale, mais qui, à cause de l'application spirituelle qui est le but principal de l'œuvre, ont subi de légères modifications.

I. Un empereur accueille dans sa cour un homme pauvre et humble qui lui promet de lui rendre six services. Le premier est de bien le servir pendant une année. Il prépare le lit du maître, reste couché en armes devant sa porte chaque nuit et a [295] un petit chien auprès de lui qui le réveille par ses aboiements chaque fois que le sommeil s'empare de lui. Le deuxième service consiste à veiller pendant une année quand les autres dorment, et de dormir quand les autres sont éveillés. Le troisième <est> de savoir juger une boisson. L'empereur fait mélanger du vinaigre, du vin et du cidre dans un gobelet et <le> lui fait donner, il goûte et dit "c'était bon, c'est bon et ce sera bon». Car le cidre deviendra bon, le vin est bon et le vinaigre était bon. Quatrièmement, il doit aller visiter tous les riches et inviter les amis de son maitre. Il invite cependant tous les ennemis et dit «c'est mieux ainsi, car ils doivent aussi devenir ses amis»; et avant que la fête ne commence, il a transformé leurs cœurs. Le cinquième service est de faire du feu sans fumée. Il place du bois séché au soleil qui s'embrase sans fumée par la chaleur. Le sixième service consiste à montrer à ceux qui veulent aller en Terre promise la bonne route par laquelle ils partiront et reviendront aisément. Il les conduit tous à la mer et dit : «Là-bas, un oiseau se tient sur un rocher, qui couve sept œufs avec grande diligence. Tant qu'il s'y tient, la mer est calme, mais s'il s'envole, alors il fera une telle tempête que personne ne pourra traverser. Mais il n'abandonne jamais le nid, si ce n'est quand un autre oiseau, qui est son ennemi, vient, souille le nid et brise les œufs, chose que ce dernier désire constamment. On peut cependant l'en empêcher si on enduit l'intérieur et l'extérieur du nid avec le sang d'un agneau ". Les pèlerins $\{320\}$ remplissent cette condition et voyagent en sûreté à 
l'aller et au retour. L'empereur récompense finalement le fidèle serviteur. Éd. lat. de I 489 Fol. chap. I7. Édition allemande également de I489 Fol. chap. 48 (où cependant il n'effectue que cinq services, au contraire de l'éd. lat. Venise 1516 in 8 , où c'est à nouveau six).

2. Un conte qui concorde avec l'entrée en matière du Diable aux trois cheveux [Teufel mit den drei goldenen Haaren] ( $\left.\mathrm{n}^{\circ} 29\right)$, mais qui apparaît aussi comme légende du roi Heinrich (Deutsche Sagen 2, $n^{\circ} 480$ ). Éd. lat. chap. 20. Éd. allemande chap. 44.

3. Un malfaiteur est saisi et doit être gracié s'il dit trois vérités que personne ne peut contester. Sur quoi il dit : "J'ai été pendant toute ma vie une mauvaise personne». Deuxièmement : "Cela ne me plaît pas [296] d'être arrivé ici par ce chemin». Troisièmement : «Si cette fois je me libère, je ne reviendrai pas volontairement ». Sur quoi il est gracié. Éd. lat. chap. 58. Éd. allemande chap. 45. Dans les Antiquités de Cambridge [Alterthümern aus Cambridge] de Robert, on raconte une légende populaire ${ }^{96}$ semblable. Artus s'égare pendant la chasse et parvient à une grotte dans laquelle habite une vieille géante avec son fils et sa fille. La mère et le fils veulent le tuer, mais la fille fait en sorte que la vieille accepte de le laisser en vie s'il est capable, le matin suivant, de dire trois vérités. Artus est bien traité, le jeune géant lui joue de la harpe. Une fois qu'Artus est en train de se reposer, l'autre le couvre d'une peau de bœuf si lourde qu'il ne peut pas bouger. Le matin suivant, Artus dit trois vérités. S’adressant en premier au fils : "Vous êtes le meilleur joueur de harpe que j'aie jamais entendu». "Cela est vrai», dit la vieille. Â celle-ci : «Vous, la sorcière la plus affreuse que j'aie jamais vue». "De nouveau, c'est vrai». En troisième, "Si un jour je partais, je ne reviendrais jamais». Cela est aussi admis et Artus est remis en liberté.

4. Le roi veut donner sa fille en mariage à celui qui la battrait à la course; en revanche, on coupera la tête au perdant. Un pauvre jeune homme relève le défi. Il lui jette d'abord une couronne de roses sur la piste, elle la ramasse et pendant qu'elle se la place sur la tête, il arrive avant elle. Alors, elle jette la couronne $\{\mathbf{3 2 I}\}$ et dépasse son adversaire. La deuxième fois, il jette une ceinture d'or, elle la ramasse et s'en ceint la taille, mais quand elle voit qu'elle a été distancée, elle la déchire en trois morceaux, devance à nouveau le jeune homme, le frappe au visage et dit : «Misérable, tu ne pourras jamais me prendre pour épouse». Alors, il jette la troisième fois une bourse dans laquelle se trouve une pomme dorée sur laquelle

96. L'expression «légende populaire» traduit l'allemand «Volkssage». 
est inscrit : «Qui joue avec moi ne se lasse jamais du jeu». Sur quoi, elle commence à jouer avec la pomme, le jeune homme atteint avant elle l'arrivée et on la marie à lui. Éd. lat. chap. 6o. Éd. allemande chap. 63. Immédiatement vient ici à l'esprit la légende d'Atalante.

5. Les deux médecins [Die beiden Ärzte]. Éd. lat. chap. 76. Éd. allemande chap. 37. Voir l'annotation du conte allemand $n^{\circ}$ II8. [297]

6. Qui est le plus paresseux, aura l'empire. Éd. lat. chap. 9I. Éd. allemande chap. 3. Voir annotation du conte allemand $n^{\circ}$ I 5 I.

7. Deux serpents, un mâle et une femelle, auxquels la vie du roi et de la reine est liée. Éd. lat. 92.

8. Facétie des trois affamés qui ne trouvent qu'un $<$ seul $>$ pain et qui conviennent que c'est celui qui fera le meilleur rêve qui le recevra. Pendant que les deux autres dorment, le troisième mange le pain et en rêve ensuite. Éd. lat. 106.

9. Un chevalier orgueilleux et arrogant tombe avec son cheval dans une fosse pour animaux, dans laquelle tombent successivement un lion, un singe et un serpent. Un pauvre bûcheron passe <dans les parages $>$ et sort d'abord les animaux les uns après les autres, ainsi qu'en dernier l'homme avec le cheval. Celui-ci <lui> promet une grande récompense, mais quand le pauvre revient plus tard, il le maltraite et le bat. Au bout d'un certain temps, celui-ci travaille de nouveau dans le bois, alors le lion lui amène chez lui des ânes richement chargés. Le pauvre, toutefois, demande publiquement si quelqu'un a perdu ces trésors; un <homme> se présente et les emporte chez lui. Une autre fois, il veut couper du bois, mais puisqu'il n'a pas de hache, le singe $<$ en $>$ ronge pour lui et tire toute une charge. En troisième lieu, le serpent lui tend avec sa gueule une pierre tricolore, noire, blanche et rouge, $\{\mathbf{3 2 2}\}$ et c'est une pierre porte-bonheur. Le roi veut l'acheter, mais il doit proposer le prix qu'elle vaut, sinon elle reviendra d'elle-même au vendeur. À cette occasion, le pauvre raconte comment le chevalier orgueilleux, qui est serviteur du roi, l'a récompensé pour l'aide qu'il lui a apportée; en punition, on pend ce dernier à la potence et son office échoit au pauvre. Éd. lat. chap. II9. Éd. allemande chap. 76. Comp. le conte souabe chez Meier $n^{\circ}$ I4 et dans le Pentamerone $3,5$.

Io. Le conte de Fortunat. Lat. chap. I20. Éd. allemande 8. Comp. le conte allemand $\mathrm{n}^{\circ}$ I22. 
II. Un serpent porte chance, mais quand il est tué par cupidité, la chance disparaît. Lat. chap. I4I. Allemand chap. 88. Voir l'annotation du conte du crapaud [Unke] $\mathrm{n}^{\circ}$ I05. [298]

I2. Un <homme> survient, moitié à cheval, moitié à pied, il emmène son pire ennemi, son meilleur ami et un ménétrier. Éd. lat. chap. I24. Éd. allemande chap. 24. Comp. l'annotation du conte allemand de La fille avisée du paysan [Kluge Bauerntochter] $\mathrm{n}^{\circ} 94$.

I3. Un roi désire le domaine d'un chevalier. Il lui dit : «Si tu ne m’apportes pas un cheval noir, un chien noir, un faucon noir et un cor noir sous huit jours, alors tu perdras ta terre». Triste, le chevalier va dans un bois; là se trouve un vieillard assis, tenant à la main un bâton qu'il lui tend avec ces mots : "Pars tout droit avec ça, ainsi tu arriveras à un château noir, là, exige, au nom de celui à qui appartient ce bâton, un cheval noir, un chien noir, un faucon noir et un cor noir. Quand tu auras tout, alors garde-toi de monter sur le cheval, de jouer du cor, ne laisse pas non plus chasser le chien ou voler le faucon, même s'ils te presseront de le faire. Rapporte ensuite le tout à ton maître, à moi toutefois tu rapporteras le bâton.» Après trois jours, le chevalier aperçoit le château; tout s'accomplit. Le roi se réjouit d'avoir obtenu <ce qu'il a $>$ exigé, lorsqu'il entend aboyer les chiens. Les serviteurs lui disent qu'un cerf est là. Alors, le roi monte sur le cheval noir, appelle le chien noir, prend le $\{323\}$ faucon noir à la main et s'accroche le cor noir autour du cou. Aussitôt qu'il aperçoit le cerf, il joue du cor et s'approche à cheval. Le cerf toutefois fonce à toute vitesse directement dans le précipice, le roi le suit, et on ne l'a jamais plus revu. D'un manscr. viennois lat. des Gesta R. Cod. univ. no ${ }^{\circ} 172$. Bl. 248 et dans l'édition allemande chap. 34. Apparait aussi au fond dans les récits de Nicolaus de I470, mais les GESTA ROMANORUM en sont a la source, communiquée par Hagen dans les Récits et facéties [Erzählungen und Schwänke] de Büsching p. I24-I26. Chez Gräße 2, 208. Comp. la légende de la mort de Dietrich de Berne.

I4. Un roi a une très belle fille, qui ne veut épouser que celui qui pourra résoudre trois tâches. Beaucoup se présentent, mais échouent. C'est alors qu'arrive un chevalier, accompagné seulement d'un serviteur et d'un cheval malade. D'abord, il doit dire combien de pieds en longueur, largeur et profondeur font les quatre éléments. [299] Le chevalier ordonne à son serviteur de se coucher, il le mesure de la tête aux pieds et répond alors : «Sept pieds en longueur, un demi-pied en largeur font les quatre éléments qui sont complètement réunis dans l'être humain ». En deuxième, <il doit dire> ce qui change la direction du vent depuis le 
nord. Il répand une poudre sur les narines de son cheval furieux, suite à quoi il se trouve guéri, il positionne ensuite sa tête renâclante vers l'est et dit "La direction de l'air s'est modifiée vers l'est, car la vie de l'animal consiste en un souffle». En troisième lieu, il doit porter des charbons ardents, sans se brûler, sur sa poitrine, <à même> la peau. Il effectue cela et si c'est possible, c'est parce qu'il porte sur lui une pierre qui a la force de protéger contre toute influence de l'eau ou du feu. Sur ce il reçoit la fille du roi. Chap. 70 ici selon le manuscrit viennois Bl. 249.

Un autre récit, bien que différent, mais basé sur un fondement semblable, est fourni par les GESTA ROMANORUM remaniés en Angleterre et pourvus de nouvelles pièces et qui sont communiqués dans la traduction de Gräße p. 230. 23I. L'empereur Andronicus présente à un chevalier accusé à tort trois questions insidieuses, auxquelles il doit répondre sous peine de mort \{324\}. I. "Combien y a-t-il du ciel jusqu'à l'enfer?" «La même distance que d'un soupir au cœur.» <2.> "Quelle est la profondeur de la mer? " "La longueur d'un jet de pierre.» 3. "Combien de bouteilles d'eau salée y a-t-il dans la mer?" "Indique d'abord les bouteilles d'eau douce, alors je dirai les autres. " Le chevalier doit, de plus, expliciter ses réponses. Il dit I. «Un soupir vient du cœur à la vitesse de l'éclair. » 2. "La pierre, parce qu'elle est lourde, tombe d'un coup au fond de la mer." 3. "Il sera temps d'estimer les bouteilles d'eau salée quand vous aurez commencé à calculer les bouteilles d'eau douce.» Que l'on compare à cela le conte allemand n ${ }^{\circ} \mathrm{I} 52$.

\section{$\{324\} /[299]$ Charles Perrault}

Les véritables recueils de contes apparaissent en France seulement à la fin du XVII ${ }^{e}$ siècle, donc après les <recueils> italiens, [300] époque à laquelle on montre un grand goût pour <le genre $>^{*}$. Nous négligeons l'opinion commune selon laquelle on considère $<$ que $>$ l'origine de ces créations ${ }^{97}$, généralement admise comme <étant> obscure, <serait> la conséquence de la connaissance des récits arabes, associée au souvenir des poèmes des trouvères et troubadours* ${ }^{* *}$ On n'a pas besoin d'objecter que la traduction par Galland des Mille et Une Nuits [Tausend und eine Nacht] parut seulement après la mort de Perrault (en 1704); la parenté des contes français

* Dont le comte de Caylus fait expressément mention dans le prologue au récit CADICHON (CABINET DES FÉES 25, <page> 409).

97. Le terme "création" traduit ici l'allemand "Dichtung".

** Bouterweck, Geschichte der Poesie 6, <page> 244. Voir Valkenaer, LETTRES SUR LES CONTES DE fÉes attribuées à PerRault et SUR L'origine de LA FÉERIE, Paris, i826. 
avec les italiens et les allemands et, en même temps, leur indépendance évidente prouve irréfutablement, ce qui ressort déjà de leur esprit, que leur contenu a été tiré de la tradition orale. Les emprunts que Dunlop (p. 408 chez Liebrecht) tente de prouver, sont tous infondés. À cela s'ajoute accidentellement une preuve externe. Scarron (né en i6Io, mort en I660) se rappelle probablement déjà avant Perrault (né en I633, mort en 1703) dans le ROMAN COMIQUE (Paris I65I, p. 78) de PEAU \{325\} D'ANE. Perrault a rédigé ses contes dans un style pur et n'a, hormis quelques détails, rien ajouté : son style est simple et naturel et, autant que le permettait la langue écrite, déjà uniformisée et polie à l'époque, il a aussi approché le ton puéril. Des expressions idiomatiques sont bien conservées, p. ex. elle allait $<\ll>$ tant que la terre put la porter $\langle »\rangle$; il vient $\langle\ll\rangle$ de douze mille lieues de là $\langle »\rangle$, ou $<\ll>$ je vais manger ma viande <»> je veux manger; et, très certainement, $<$ c'est $>$ de la tradition orale que provient, dans Barbebleue [Blaubart], la question et la réponse <«> Anne, ma sœur Anne, ne vois tu rien venir? <»> $<«>$ Je ne vois rien que le soleil qui poudroie, et l'herbe qui verdoie ${ }^{98} .\langle »>$ $<$ C'est à ces $<$ mérites $>$ que le livre doit sans aucun doute d'avoir perduré jusqu’à notre époque.

I. Les femmes sages [Die weisen Franen] (LES FÉES). Dans le PENTAMERONE 3, IO et 4, 7, chez nous $\mathrm{n}^{\text {os }}$ I3 et 24 . Le français est le plus pauvre.

2. La belle dormant dans le bois [Die schlafende Schöne im Walde] (LA BELLE AU BOIS DORMANT). PENTAMERONE Soleil et Lune [Sonne und Mond] 5, 5 , chez nous Rose d'épine [Dornröschen] n ${ }^{\circ}$ 50. [30I]

3. Barbebleue [Blaubart] (LA BARBE BLEUE). En allemand $\mathrm{n}^{\circ} 46$ L'oiseau de Fitcher [Fitchers Vogel], cependant bien divergent; en italien rien de semblable.

4. Le chaperon rouge [Rothkäppchen] (LE PETIT CHAPERON ROUGE). Allemand $\mathrm{n}^{\circ} 26$.

5. Le chat botté [Der gestiefelte Kater] (LE CHAT BOTTË). PENTAMERONE Galiuso ${ }^{99}$ 2, 4. Straparola II, I. Fragment n ${ }^{\circ} 4$.

6. Cendrillon [Aschenputtel] (CENDRILLON). Plus plat que dans le PENTAMERONE I, 6 et que l'allemand $\mathrm{n}^{\circ}$ 2I. Quelle importance a le motif, complètement absent en français, selon lequel les méchantes sœurs trompent un instant le fils du roi en se mutilant violemment les pieds pour porter des chaussures, mais sont trahies par des colombes.

98. Les citations de Perrault mises ici entre guillemets sont données en français par les Grimm eux-mêmes.

99. Nom tel que le donnent les Grimm; chez Basile : Cagliuso. 
7. Riquet avec la houppe [Riquet mit dem Schopf] (RIQUET À LA HOUPPE). Pourrait en premier compter comme une simple invention. Il ne contient rien d'autre que l'idée qu'un homme laid mais spirituel peut accorder de l'esprit à une fille, et qu'une fille belle mais stupide <accorder> de la beauté à un homme difforme, à condition de l'aimer. Aussi trouve-t-on ici des tournures amusantes, épigrammatiques et un dialogue finement piquant. En italien et en allemand, rien de semblable.

8. Le petit poucet [Der kleine Däumling] (LE PETIT POUCET). En gros, le conte allemand Hänsel ${ }^{\circ}$ I5. Dans le PENTAMERONE 5, 8. \{326\} Le poucet [Der Däumling] n'est ici pas aussi caractéristique ${ }^{100}$ que dans les deux contes allemands $\mathrm{n}^{\text {os }} 37$ et 45 .

Ces huit pièces, Perrault les publia avant (?), Paris I697 in I2 sous le vieux titre emprunté à un fabliau CONTES DE MA MERE L'OYE, et sous un deuxième, HisTOIRES OU CONTES DU TEMPS PASSÉ. Dans les éditions suivantes, s'en ajoutèrent encore trois ${ }^{*}$. [302]

9. Peau d'âne [Eselshaut] (PEAU D'ÂNE). PENTAMERONE L'ourse [Bärin] $(2,6)$, en allemand Toutes-fourrures [Allerlei-Rauh] ( $\mathrm{n}^{\circ}$ 65).

Io. La fille de roi avisée [Die kluge Königstochter] (L'ADROITE PRINCESSE) ${ }^{\mathrm{IO}}$. Dans le PENTAMERONE SAPIA LiCCARDA $(3,4)$.

II. Les souhaits ridicules [Die lächerlichen Wünsche] (LES SOUHAITS RIDICULES) en vers. Contient la dernière partie du conte allemand du pauvre et $d u$ riche [Der Arme und der Reiche] ( $\left.\mathrm{n}^{\circ} 87\right)$.

\section{\{326\}/[302] Comtesse d'Aulnoy}

La comtesse d'Aulnoy (née en I650, morte en I705), connue aussi pour d'autres travaux, vécut à la même époque que Perrault. Elle doit avoir écrit ses contes, du moins une partie de ceux-ci, après la publication des siens, donc dans les dernières années de sa vie, puisque, dans LA CHATTE BLANCHE

IOO. Le terme «caractéristique» traduit l'allemand «eigenthümlich».

* Dans quelques éditions encore un quatrième <conte>, concrètement GRISELDIS en vers. Dans la magnifique <édition> parisienne 1782 in I2 et dans le CABINET DES FÉES I, il y a de ce fait douze pièces; seulement GRISELDIS n'est pas un conte, mais une nouvelle fameuse <tirée> de Boccace et est de ce fait omise avec raison dans d'autres éditions. Nicheron (MÉMOIRES POUR SERVIR À L'HISTOIRE DES HOMMES ILLUSTRES 33, <page> 287) suppose que Perrault est né vers l'an i626 et allègue GRISELDIS, NOUVELLE AVEC LE CONTE DE PEAU D'ASNE ET CELUI DES SOUHATTS RIDICULES, deuxième édition Paris I694 in I2, avec la remarque que tout cela aurait été rédigé en vers.

IoI. Les Grimm placent donc ce conte de Mme L'Héritier dans leur liste de Perrault, comme le font les éditions du XVIII ${ }^{\mathrm{e}}$ siècle et Le Cabinet des fées dont ils tirent manifestement leurs informations. 
( $\mathrm{n}^{\circ}$ 19), elle mentionne la PEAU D'ÂNE, la BELLE AU BOIS DORMANT et <le> CHAT BOTTÉ, en pensant pour les deux derniers très certainement aux contes de Perrault. Pourtant, elle ne l'a pas imité, son recueil est à la fois moins bon et meilleur. Moins bon, dans le sens où les traditions y sont moins conservées et où elle y a mélangé des ajouts, des développements, des vers et des considérations morales, et où, de manière générale, la matière est traitée plus arbitrairement. Les traditions, toutefois, sont à la base d'une grande partie de ces contes, comme de ceux de Perrault $\{327\}$ et les autres, complètement inventés, s'en différencient facilement par manque de consistance. Une preuve assez étrange en est un des plus beaux <contes>, L'oiseau bleu [Der blaue Vogel], puisqu' on le retrouve de façon indéniable dans les poèmes ${ }^{\mathrm{IO2}} \mathrm{de}$ Marie de France, qui vivait déjà au début du XIII siècle; c'est le lai d'Yonec [Lai von Ywenec] (272-313), une légende gauloise, qui a donc perduré sur terre française jusqu'au XVIII ${ }^{\mathrm{e}}$ siècle. Seulement les trois derniers <contes> $\left(\mathrm{n}^{\mathrm{os}} 22,23,24\right)$ sont pris chez Straparola, dont les contes ont été introduits en France en traduction : on voit facilement qu'ils ont été modifiés et pour quelle raison. On ne peut pas qualifier de maladroite la manière d'Aulnoy, au contraire, on y voit une main aisée, déjà exercée; nombre de choses sont aimablement racontées et beaucoup sont exprimées de façon naïve et puérile; pourtant, les contes n’ont pas rencontré l'adhésion générale, parce qu'ils convenaient seulement à des enfants de la classe supérieure à laquelle appartenait l'auteur. Il y a trop d'ornements et de préciosité, probablement aussi de cette sentimentalité française [303]; on y perçoit le raffinement <extrême> et la distinction de l'époque de Louis XIV; il y manque par contre quelque naturel et quelque fraîcheur, ou la simplicité et, si on veut bien ne pas mal interpréter l'expression, l'air bourgeois qui transparaît, à côté de tous les faits merveilleux, dans les contes authentiques ${ }^{103}$. Par contre, ils sont meilleurs que ceux de Perrault, dans le sens où ils se fondent sur une tradition plus riche et plus belle; aussi, du moins à première lecture, est-on disposé à considérer comme un mérite l'entrelacement plus artificiel des événements, le polissage et le remaniement, souvent habile, qui les rapproche du petit roman. Si ce qui importait en poésie était seulement le prétendu embellissement esthétique, alors on ne comprendrait pas pourquoi les contes de Perrault, moins charmants, l'ont emporté. Nous les énumérons un par un ${ }^{*}$ et indiquons les ressemblances avec d'autres; un extrait serait superflu pour un livre <si> souvent réimprimé.

IO2. Le terme "poèmes» traduit l'allemand «Gedichte».

I03. L'adjectif "authentique» traduit l'allemand "echt».

* Selon l'édition du CABINET DES FÉES, Paris, 1785, vol 2. 
I. Gracieuse et Percinet [Gracieuse und Percinet]. Ressemble à un conte italien du PENTAMERONE 5, 4. Visiblement rajouté : le palais des fées de Percinet. $\{\mathbf{3 2 8}\}$

2. La belle aux cheveux d'or [Die schöne mit dem Goldhaar] (LA BELLE AUX CHEVEUX D'OR). Dans le PENTAMERone Corvetto $(3,7)$, en allemand Fernand-le-fidèle [Ferenand getrü] ( ${ }^{\circ}$ I26). Le cheval blanc qu'y crée Rath est ici le petit chien Cabriolle. Fin et assez dénué d'ajouts.

3. L'oiseau bleu [Der blaue Vogel] (L'OISEAU BLEU). Visiblement en relation, comme déjà indiqué, le lai d'Yonec [Lai von Ywenec] du XIII ${ }^{\mathrm{e}}$ siècle. L'allemand Alouette [Löweneckerchen] ( ${ }^{\circ}$ 88), mais seulement à partir du moment où le fils du roi, transformé en colombe, doit s'envoler.

4. Le lutin [Der Kobold] (LE PRINCE LUTIN). A un bon fondement. Le prince sauve un serpent sans savoir qu'une fée se cache sous cette apparence; celle-ci lui offre toutes les qualités d'un lutin. Entre autres, elle lui donne aussi un chaperon rouge (bonnet de brouillard) grâce auquel il peut se rendre invisible. [304]

5. PRINTANiÉRE. Inventé, en majeure partie; certains traits sont authentiques, p. ex. la fille du roi, qui doit vivre cachée pendant vingt ans, regarde peu avant le terme à travers une ouverture si petite qu'à peine une aiguille peut y passer, et devient triste. La façon dont les arbres se montrent serviables est également jolie.

6. ROSETTE. En allemand, le conte de La fiancée blanche et la fiancée noire [Die weisse und schwarze Braut] ( $\mathrm{n}^{\circ}$ I35), mais très différent. Le trait selon lequel la véritable épouse est jetée à la mer, alors qu'elle dort dans son lit, mais qu'heureusement elle ne peut pas couler, parce que $<$ le lit $>$ est rempli de plumes miraculeuses, est inhabituel mais beau; cependant, l'allemand est certainement supérieur, avec l'esprit s'élevant de l'eau comme un oiseau. À comparer, la légende anglo-saxonne du roi Scyld Scafing (de sceaf, ancien allem. scoup, gerbe), qui arrive à la nage tout en dormant sur une botte de paille; voir Göttinger gel. Anzeigen I823 n ${ }^{\circ} \mathrm{I}$.

7. La branche d'or [Der goldene Ast] (LE RAMEAU D'OR). Peu de bonnes choses et, autrement, beaucoup d'êtres féeriques et de bergers, suivant la mode de cette époque.

8. L'oranger et l'abeille [Der Orangenbaum und die Biene] (L'ORANGER ET L'ABEILLE). La première partie, le séjour chez l'homme sauvage et les amours secrets, est agencée de façon moderne; à partir du moment, cependant, où les deux veulent s'enfuir ensemble, le \{329\} conte est authentique et beau et visiblement apparenté aux <contes> allemands 
du Bien-aimé Roland [Liebsten Roland] ( $\mathrm{n}^{\circ}$ 56) et des Deux enfants royaux [beiden Königskinder] ( ${ }^{\circ}$ II 3 ). Parmi les transformations pendant la fuite, la dernière est originale et pertinente, la fille transforme le bien-aimé en un oranger <et se transforme> elle-même en abeille par laquelle la sorcière, qui les poursuit, est piquée jusqu'au sang, de manière à la faire fuir.

9. La bonne petite souris [Die gute kleine Maus] (LA BONNE PETITE SOURIS). Dans la manière d'agir de la souris et de tourmenter le méchant roi, ce conte, par ailleurs original, a cependant une ressemblance avec le Bousier, la souris et Heinchen [Mistkäfer, Maus und Heinchen] dans le PENTAMERONE $(3,5)^{\text {ro4. }}$.

Io. Le bélier [Der Widder] (LE MOUTON)*. Dans le fond, la légende d'Amour et Psyché est à la base quand même, même si tout a été fortement [305] transformé. Des contes allemands semblables sont cités dans l'annotation du n 88.

II. FINETTE CENDRON. Tout d'abord, <y est apparenté> le conte des Frères et sours rejetés [Verstoßene Geschwister], qui sont ici non pas deux mais trois et qui sont les enfants du roi, en allemand $\mathrm{n}^{\circ} \mathrm{i} 5$, dans le PENTAMERONE 5, 8; ensuite, cependant, y est aussi relié Cendrillon [Aschenputtel] dans le PENTAMERONE I, 6, allemand $\mathrm{n}^{\circ}$ 2I, chez Perrault $\mathrm{n}^{\circ}$ 6. L'originalité résulte aussi précisément de cela, puisque chaque <version $>$ a son caractère ${ }^{105}$. Est joli dans la première partie le trait selon lequel les trois fugitives trouvent un gland quelles plantent dans la terre et qu'elles arrosent matin et soir, dans l'espoir de pouvoir un jour, du haut du petit arbre, $<$ une fois $>$ qu'il aurait grandi, regarder autour d'elles.

I2. Fortuna (FORTUNÉE). A seulement des détails authentiques, et non un fondement authentique.

13. BABIOLE. Complètement inventé comme le suivant.

I4. Le nain jaune [Der gelbe Zwerg] (LE NAIN JAUNE)**.

I04. Titre chez Basile : «Lo scarafone, lo sorece e lo grillo»; Decroisette : «Le scarabée, le rat et le grillon».

* Ce <conte> et les deux suivants sont enchâssés dans la nouvelle de PONCE DE LEÓN.

I05. Le terme "caractère" traduit l'allemand "Eigenthümlichkeit».

** $\mathrm{N}^{\text {os }}$ I4 et $\mathrm{I} 5$ se trouvent dans un récit, FERNAND DE TOLĖDE. 
15. Le serpent vert [Die grüne Schlange] (LE SERPENT VERT $\left.{ }^{100}\right)$. Apparenté avec le conte d'Amour et Psyché; pour le reste, bien différent; voir annot. au $\mathrm{n}^{\circ} 88$.

I6. CARPILLON. Pas vraiment un conte.

17. La grenouille bienfaisante [Der wohltätige Frosch] (LA GRENOUILLE BIENFAISANTE). Invention sans valeur. $\{330\}$

18. La biche dans le bois [Die Hindin im Wald] (LA BICHE AU BOIS). Un beau conte qui a une parenté éloignée avec l'allemand $n^{\circ}$ II. La fille du roi n'a pas le droit de voir la lumière du soleil avant sa quinzième année, peu avant le terme, elle l'aperçoit et est transformée en une biche. Elle recouvre son aspect humain seulement lorsque son bien-aimé, sans le savoir, la blesse pendant une chasse.

19. La chatte blanche [Die weiße Katze] (LA CHATTE BLANCHE)*. C'est le conte allemand des Trois plumes [Drei Federn] ( ${ }^{\circ}$ 63) et du Chatton [Kätzchen] ( $\mathrm{n}^{\circ}$ I06), entrelacé avec Rumpelstilzchen ( $\left.{ }^{\circ} 55\right)$.

20.Fortunatus (BELLE-BELLE OU LE CHEVALIER FORTUNE). En allemand, Six à travers le monde [Sechse durch die Welt] ( $\left.\mathrm{n}^{\circ} 7 \mathrm{I}\right)$, dans le PENTAMERONE le Sot [Dummling] $(3,8)$. [306]

2I. Le couple de colombes [Das Taubenpaar] (LE PIGEON ET LA COLOMBE). Très élaboré et modernisé. L'entrée en matière est certainement authentique. La fille du roi est cachée et ne doit pas sortir de la maison pour ne pas tomber entre les mains du géant. Quand elle entend cependant son petit agneau chéri crier de peur face au loup, elle oublie l'avertissement et sort en courant. Le géant la met alors, avec le loup, l'agneau et encore d'autres animaux, dans un sac, et, puisqu'il doit se battre avec un autre géant, il lance entre-temps le sac sur un arbre. Alors, la fille coupe le sac avec des ciseaux, se libère, prend avec elle le petit agneau et les autres animaux et laisse seulement le méchant loup à l'intérieur.

22. La belle avec l'étoile [Die Schöne mit dem Stern] (LA PRINCESSE BELLEÉTOILE). Directement de Straparola 4, 3.

23. Prince cochon [Prinz Schwein] (LE PRINCE MARCASSIN). Straparola 2, I. 24. Le dauphin [Der Delphin] (LE DAUPHIN). Straparola 3, I.

IO6. Les Grimm donnent en français comme titre "LE SERPENT VERT», au lieu du titre original, «Serpentin vert».

* Celui-là, comme tous <les contes> suivants, dans un récit, LE GENTILHOMME BOURGEOIS. 


\section{\{33I\}/[307] Imitateurs}

Les contes qui parurent après la comtesse d'Aulnoy au début du XvıII ${ }^{\mathrm{e}}$ siècle en assez grand nombre sont tous très inférieurs et relèvent presque toujours de vaines fantaisies, sans substance véritable. Notamment, $\mathrm{ce}^{\mathrm{107}}$ que composèrent <comme étant des> contes la comtesse de Murat (morte en I7I6) dans le CABINET DES FÉES vol. I, la comtesse d'Auneuil (morte en I700), idem vol. 5, le Sieur de Preschac (né en I676), idem vol. 5, est un mélange d'êtres pseudo-orientaux à de modernes histoires d'amour entre pâtres, sans vraie consistance; les figures sont dépourvues de vie et d'originalité. On ne peut pas juger plus avantageusement les prétendus contes du comte d'Hamilton (né vers I656, mort en I720), idem vol. 20 et de Monsieur de Moncrif (né en I687, mort en I770), idem vol. 26. Parmi les récits de Mademoiselle de La Force (née vers I650, morte en I724), idem vol. 6, seul le deuxième, PERSINETTE, mérite d'être mentionné; c'est PETROSINELLA dans le PENTAMERONE 2, I, mais selon une tradition très appauvrie et altérée. L'annotation d'un autre récit dit d'ailleurs explicitement que seul ce dernier (intitulé L'ENCHANTEUR) est tiré d'un livre, mais que tout le reste est invention de l'auteur. [307] Dans les contes de Mademoiselle L'heritier ${ }^{108}$ (née en 1667, morte en 1737), idem vol. I2, un seul, RICDIN-RICDON, a un fondement authentique. Par son introduction, il correspond au conte allemand des Fileuses [Spinnerinnen] ( $\mathrm{n}^{\circ}$ I4), et se change ensuite en Rumpelstilzchen ( $\left.\mathrm{n}^{\circ} 55\right)$; mais ici aussi, la tradition s'est élargie en un petit roman, <cela> moyennant un dommage sensible. Dans le vol. 5, il y a encore un recueil titré LES ILLUSTRES FÉES, d'un auteur anonyme; il faut y noter deux pièces : BLANCHEBELLE, avec un écho au conte allemand de La fiancée noire et la fiancée blanche [Die schwarze und die weisse Braut] ( $\left.\mathrm{n}^{\circ} \mathrm{1} 35\right)$, et Le Prince Guerini, directement de Straparola (5, I) le Cadeau des trois animaux [Geschenk der drei Thiere]. Les histoires merveilleuses (FÉERIES NOUVELLES) du comte de Caylus (idem vol. 24), qui écrivait durant la première moitié du XVIII ${ }^{\mathrm{e}}$ siècle, sont pour nous vaines et sans valeur, et seulement dans l'une, Tourlou et Rirette, se trouve une partie d'un conte, intitulé L'oiseau jaune et inséré en tant que fable morale. Il contient l'entrée en matière $\{332\}$ des Deux frères $\left(\mathrm{n}^{\circ} 60\right)$. Une magicienne est transformée en un oiseau jaune et capturée. Un riche l'achète à l'homme qui l'a emprisonnée, et comme il trouve écrites sur l'aile droite les paroles «Celui qui mange ma tête devient roi; celui qui mange mon cœur, a chaque matin, dès son réveil, cent pièces d'or ", ainsi il se fait

I07. "Ce» traduit ici l'allemand «was», utilisé peut-être un peu dédaigneusement par les Grimm. I08. Les Grimm écrivent le nom de L'Héritier sans accent. 
rôtir l'oiseau par la femme du pauvre homme. Celle-ci donne cependant par hasard la tête et le cœur à manger à ses deux garçons qui par la suite fuient la colère du trompé. L'un est assassiné à cause de sa richesse, l'autre arrive dans un royaume où l'on est précisément divisé sur le choix d'un roi et où l'on attend des signes. Comme une colombe se place sur sa tête, il est ainsi élu, mais il est assassiné lors d'un soulèvement à cause de son mauvais gouvernement. On en tire la moralité suivant laquelle chacun devrait rester dans son rang, <moralité>, cependant, pour laquelle ce développement a certainement été ajouté. Un recueil de contes, NOUVEAUX CONTES DE FÉES, dont l'auteur n'est pas connu, parut en l'an I7I8 et à nouveau en I73I, et fut, comme les deux éditions s'étaient faites rares, réimprimé dans le CABINET $D E S$ FEES vol. 3I. Parmi les neuf pièces qui le constituent, seulement trois $\left(\right.$ la i $^{\text {re }}$, la $5^{\text {e }}$ et la $9^{\mathrm{e}}$ ) ont un fond ${ }^{\text {109 }}$ consistant et peuvent provenir d'une tradition vivante. [308]

I. La petite rainette [Der kleine Laubfrosch] (LA PETITE GRENOUILLE VERTE). Un roi malade exige un oiseau miraculeux, son fils part à sa recherche et arrive à un puits où une rainette le renseigne. Elle lui donne un grain de sable et lui dit de le jeter devant le château auquel il arrivera; alors, tout ce qu'il y verra s'endormira. Ensuite, il devra entrer, prendre le plus beau cheval de l'écurie et rentrer à cheval rapidement. Cependant, comme le prince voit une selle à côté du cheval, il veut d'abord la mettre <sur lui > ; alors, tout se réveille et il ne l'obtient pas. Deuxièmement, la grenouille lui donne un grain d'or, il doit faire sortir du château une vierge qui y est endormie, mais celle-ci lui demande d'abord de pouvoir mettre une jupe; alors, tout se réveille à nouveau. Troisièmement, la grenouille lui donne un grain de diamant, il doit à présent rapporter l'oiseau miraculeux y compris la branche sur laquelle il dort. Cela réussit et $\{333\}$ le roi malade est guéri. En outre, d'autres choses sont ajoutées, mais elles ne semblent pas authentiques. Le tout présente une parenté avec le conte allemand de L'oiseau d'or [der goldene Vogel] ( ${ }^{\circ}$ 57) et est seulement plus pauvre.

2. Rouge, blanc et noir [Roth, weiss und schwarz] (INCARNAT, BLANC ET NOIR). Un roi marche pendant l'hiver et voit un corbeau tomber dans la neige et tacheter de sang la blancheur de la neige. Alors, il désire une femme aussi blanche que la neige, aussi rouge que le sang et aux cheveux aussi noirs que le plumage du corbeau. Une voix lui crie que beaucoup plus loin il trouvera un arbre; il devra en cueillir trois pommes sans les

I09. Le français «fond» traduit l'allemand « Grund». 
ouvrir avant d'être de retour à la maison. Il se met en route, cueille les pommes, mais, sur le chemin du retour, il ne peut résister à la curiosité. Il ouvre l'une, il en sort alors une beauté qui le regarde méchamment et disparaît. Il ouvre encore la deuxième et a la même apparition. La troisième, il la garde et l'ouvre seulement à la maison. Il en sort une jeune fille, aussi blanche, rouge et noire qu'il l'avait souhaitée avec laquelle il se marie et vit heureux. Un jour, en son absence, la vieille et méchante belle-mère fait tuer la jeune reine et jeter son corps dans une tranchée du château. Quand le roi revient, elle parvient à faire en sorte qu'il prenne une autre femme pour épouse. Mais il est triste et un jour, regardant par la fenêtre, [309] il aperçoit un poisson miraculeux, moucheté de blanc, rouge et noir. Il le demande, mais la vieille le fait attraper et préparer pour la fausse reine. Sur ce, pousse devant la fenêtre, sans avoir été semé ni planté, un arbre des trois mêmes couleurs. La vieille le fait brûler, mais, <à partir> des cendres, surgit un beau château, de rubis rouges, de perles blanches et d'émail noir. Personne d'autre que le roi ne peut en ouvrir la porte $<$ et il $>$ y retrouve vivante la vraie reine. On reconnait le conte des Trois citrons du PENTAMERONE $(5,9)$, seulement plus lacunaire et moins consistant, et c'est précisément pour cette raison qu'il n'est pas emprunté à ce dernier. Son entrée en matière l'apparente plus précisément au Corbeau du PENTAMERONE $(4,9)$.

3. Prince arc-en ciel [Prinz Regenbogen] (LE PRINCE ARC-EN-CIEL). A quelques traits pleins de vérité et doit être rapproché du conte allemand de L'alouette [Löweneckerchen] $\{334\}$ ( $\mathrm{n}^{\circ}$ 88) et de l'italien 5, 3 et 4 dans le PENTAMERONE.

Des contes qu’a insérés Madame de Beaumont (née en I7II) dans son Livre pour enfants [Kinderbuch] (MAGASIN DES ENFANS), seul celui sur La jeune fille et l'animal (dans la cinquième conversation) est ici pertinent; il est apparenté à L'alouette [Löweneckerchen] ( $\mathrm{n}^{\circ}$ 88); les autres sont des fables morales, probablement inventées par elle. Les CONTES de Madame de Villeneuve contiennent quelques contes qui sont cités pour les ${ }^{\text {os }} 24$ et 88 d'après la traduction dans La jeune Américaine ou Le passe-temps sur mer [Die junge Amerikanerin oder Verkürzung müßiger Stunden auf dem Meer $]^{\text {I10 }}$ (Ulm I765).

IIo. Le titre de la traduction allemande mentionnée par les Grimm ne correspond pas exactement au titre courant en français : La jeune Américaine ou Les contes marins (1740). 


\section{$\{334\} /[309]$ Espagne}

Sur l'existence de contes, il ne peut pas y avoir de doutes. Un passage chez Cervantès qui en parle est mentionné ci-dessus dans les "Témoignages», et un fragment de conte de géant chez Calderon est indiqué dans les annotations du $\mathrm{n}^{\circ}$ II2. La fille du roi enchantée, dans une ancienne romance espagnole chez Diez p. 177. En outre, un passage de la comédie ${ }^{\mathrm{III}} C^{\prime}$ 'est pire que ce que c'était [Es ist schlimmer als es war] (trad. de Malsburg I, 335) semble fondé sur un conte populaire ${ }^{\mathrm{II}}$.

\section{Bibliographie}

Adam Jean-Michel et Heidmann Ute, Le texte littéraire. Pour une approche interdisciplinaire, Louvain, Academia Bruylant, 2009.

Arellano Ignacio, Francisco de Quevedo. Los sueños, édité par Ignacio Arellano, Madrid, Cátedra, I99I.

BEER Johann, Jucundi Jucundissimi, wunderliche Lebensbeschreibung, Nürnberg, Johann Hoffmann, I680.

Corominas Joan, Breve diccionario etimológico de la lengua castellana, Madrid, Gredos, 2000 [ $\mathrm{I}^{\mathrm{re}}$ éd. I96r].

Decroisette Françoise, Giambattista Basile. Le conte des contes, traduction du napolitain de Françoise Decroisette, Strasbourg, Circé, 2002 [Ire éd. 1995].

Grimm Jacob et Grimm Wilhelm, Kinder- und Hausmärchen gesammelt durch die Brüder Grimm, Berlin, fac-similé de la première édition en deux volumes de I8I2 et I8I5, selon l'exemplaire manuscrit du Brüder GrimmMuseum Kassel avec toutes les corrections et les ajouts manuscrits des Frères Grimm, transcriptions et commentaires établis en collaboration avec Ulrike Marquardt, par Heinz Rölleke, Göttingen, Vandenhoeck \& Ruprecht, 1986.

—, Kinder-und Hausmärchen gesammelt durch die Brüder Grimm. Premier volume. Deuxième édition revue et augmentée, Berlin, G. Reimer, I819.

-, Kinder- und Hausmärchen gesammelt durch die Brüder Grimm. Dritter Band, Göttingen, Dieterichische Buchhandlung, I822.

III. Le terme "comédie» traduit l'allemand "Lustspiel».

II2. L'expression «conte populaire» traduit l'allemand «Volksmärchen». 
—, Kinder-und Hausmärchen gesammelt durch die Brüder Grimm. Premier volume. Grande édition. Troisième édition revue et augmentée, Göttingen, Dieterichische Buchhandlung, I837.

—, Deutsches Wörterbuch, München, Hirzel, I854, I6 volumes en 32 volumes partiels.

—, Kinder- und Hausmärchen gesammelt durch die Brüder Grimm. Troisième volume. Troisième édition, Göttingen, Dieterichsche Buchhandlung, I856.

Heidmann Ute, «La Barbe bleue palimpseste. Comment Perrault recourt à Virgile, Scarron et Apulée, en réponse à Boileau ", Poétique, n 154 , 2008, p. I6I-I82.

—, "Comment faire un conte moderne avec un conte ancien? Perrault en dialogue avec Apulée et La Fontaine», Littérature, nº 153, 2009, p. 19-35.

-, "La (re)configuration des genres dans les littératures européennes. L'exemple des contes", Problèmes de la théorie des genres littéraires. Colloquium Helveticum, $\mathrm{n}^{\circ}$ 40, 2009, p. 9I-I04.

—, "Enjeux d'une comparaison différentielle et discursive. L'exemple de l'analyse des contes", Cahier voor Literatuurwetenschap, $\mathrm{n}^{\circ}$ 2, 2010, p. 27-40.

—, "Intertextualité et dialogicité des contes», dans U. Heidmann et J.-M. Adam, Textualité et intertextualité des contes. Perrault, Apulée, La Fontaine, Lhéritier..., Paris, Éditions Classiques Garnier, 20ı,, p. 3I-I52.

—, "Perrault en dialogue avec Apulée, Fénelon et Lhéritier : Le Petit Chaperon rouge palimpseste", dans C. Badiou-Monferran (éd.), Il était une fois l'interdisciplinarité. Approches discursives des Contes de Perrault, Louvain-la-Neuve, Academia Bruylant, 20I0, p. 85-103.

—, "Quel apport du comparatisme pour l'étude des cultures? L'exemple du Petit Chaperon rouge», dans A. Dominguez Leiva, S. Hubier, P. Chardin et D. Souiller (éds), Etudes culturelles, anthropologie culturelle et comparatisme, Dijon, Éditions du Murmure, 20IO, p. I35-I48.

—, "Expérimentation générique et dialogisme intertextuel : Perrault, La Fontaine, Apulée, Straparola, Basile», Féeries, no 8, 20II, p. 45-69.

—, «Tisserandes fatales (Apulée) et fées de cours (Perrault) : Le sort difficile d'une belle "née pour être couronnée" ", dans M. Hennard Dutheil de la Rochère et V. Dasen (éds), Des Fata aux fées : regards croisés de l'Antiquité à nos jours, Lausanne, Études de lettres, 20II, p. 205-220.

Krause Friedhilde (éd.), Die Bibliothek der Brüder Grimm. Annotiertes Verzeichnis des festgestellten Bestandes, élaboré par Ludwig Denecke et Irmgard Teitge, Stuttgart, Hirzel, I989. 
Meineke August, Strabonis Geographica, édité par August Meineke, Leipzig, Teubner, I969 [I ${ }^{\text {re }}$ éd. I877], 3 volumes.

Müller Johannes von, Sämmtliche Werke. Io. Theil. Historische Kritik, édité par Johann Georg Müller, Tübingen, Gotta, I8II.

Pirovano Donato, Giovan Francesco Straparola. Le piacevoli notti, édité par Donato Pirovano, Rome, Salerno, 2000, 2 volumes.

RaK Michele, Basile. Lo cunto de li cunti, édité par Michele Rak, traduction italienne de Michele Rak, Milano, Garzanti, 1999 [ ${ }^{\text {re éd. }}$ 1998].

Rimasson-Fertin Natacha, Contes pour les enfants et la maison. Collectés par les Frères Grimm, édités et traduits par Natacha Rimasson-Fertin, Paris, José Corti, 2009, 2 volumes.

Rölleke Heinz, Brüder Grimm. Kinder-und Hausmärchen, dernière édition avec les annotations originales, par Heinz Rölleke, Stuttgart, Reclam,

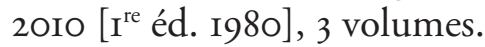

—, Die Märchen der Brüder Grimm. Eine Einführung, Stuttgart, Reclam, 2010 [Iréd. I992].

Robertson Donald Struan, Apulée. Les Métamorphoses, texte établi par Donald Struan Robertson et traduit par Paul Vallette, Paris, Les Belles Lettres, 2002 [ I $\mathrm{I}^{\mathrm{re}}$ éd. 1940-1945], 3 tomes.

Sennewald Jens E., Das Buch, das wir sind. Zur Poetik der "Kinder und Hausmärchen gesammelt durch die Brüder Grimm ", Würzburg, Könighausen \& Neumann, 2004.

Solmsen Friedrich, Hesiodi Theogonia, Opera et dies, Scutum, édité par Friedrich Solmsen, Oxford, Clarendon Press, 1970. 